OPEN ACCESS

Edited by:

Simone Brogi,

University of Pisa, Italy

Reviewed by:

Angel Diaz-Ortiz,

University of Castilla La

Mancha, Spain

Carlos Alberto Manssour Fraga, Institute of Biomedical Sciences,

Federal University of Rio de

Janeiro, Brazil

Jean Jacques Vanden Eynde,

University of Mons, Belgium

${ }^{*}$ Correspondence:

Zi-Hua Jiang

zjiang@lakeheadu.ca

Sheng-Tao Yuan

cpuYuanst@163.com

Jiang-Miao Hu

hujiangmiao@mail.kib.ac.cn

tThese authors have contributed equally to this work

Specialty section:

This article was submitted to Medicinal and Pharmaceutical

Chemistry,

a section of the journal

Frontiers in Chemistry

Received: 13 March 2019

Accepted: 28 May 2019

Published: 18 June 2019

Citation:

Zi C-T, Gao $Y$-S, Yang L, Feng $S-Y$,

Huang $Y$, Sun $L$, Jin $Y, X \cup F-Q$, Dong F-W, Li Y, Ding Z-T, Zhou J,

Jiang Z-H, Yuan S-T and Hu J-M

(2019) Design, Synthesis, and

Biological Evaluation of Novel

Biotinylated Podophyllotoxin

Derivatives as Potential Antitumor

Agents. Front. Chem. 7:434

doi: 10.3389/fchem.2019.00434

\section{Design, Synthesis, and Biological Evaluation of Novel Biotinylated Podophyllotoxin Derivatives as Potential Antitumor Agents}

\author{
Cheng-Ting Zi ${ }^{1,3,4 t}$, Ying-Sheng Gao ${ }^{2+}$, Liu Yang ${ }^{1+}$, Shu-Yun Feng ${ }^{2}$, Yue Huang ${ }^{2}$, Li Sun ${ }^{2}$, \\ Yi Jin ${ }^{4}$, Feng-Qing Xu ${ }^{1}$, Fa-Wu Dong ${ }^{1}$, Yan Li ${ }^{1}$, Zhong-Tao Ding ${ }^{4}$, Jun Zhou ${ }^{1}$, \\ Zi-Hua Jiang ${ }^{5 *}$, Sheng-Tao Yuan ${ }^{2 *}$ and Jiang-Miao Hu ${ }^{1 *}$
}

\begin{abstract}
${ }^{1}$ State Key Laboratory of Phytochemistry and Plant Resources in West China, and Yunnan Key Laboratory of Natural Medicinal Chemistry, Kunming Institute of Botany, Chinese Academy of Sciences, Kunming, China, ${ }^{2}$ Jiangsu Key Laboratory of Drug Screening and Jiangsu Center for Pharmacodynamics Research and Evaluation, China Pharmaceutical University, Nanjing, China, ${ }^{3}$ Key Laboratory of Pu-er Tea Science, College of Science, Ministry of Education, Yunnan Agricultural University, Kunming, China, ${ }^{4}$ Key Laboratory of Medicinal Chemistry for Nature Resource, School of Chemical Science and Technology, Ministry of Education, Yunnan University, Kunming, China, ${ }^{5}$ Department of Chemistry, Lakehead University, Thunder Bay, ON, Canada
\end{abstract}

Podophyllotoxin has long been used as an active substance for cytotoxic activity. Fourteen novel biotinylated podophyllotoxin derivatives were designed, synthesized, and evaluated for cytotoxic activity for this study. The synthesized compounds were evaluated for cytotoxic activity in the following human cancer cell lines, SW480, MCF-7, A-549, SMMC-7721, and HL-60 by MTT assay. Most of them exhibited potent cytotoxic effects and compound $\mathbf{1 5}$ showed the highest cytotoxic activity among the five cancer cell lines tested, having its $\mathrm{IC}_{50}$ values in the range of 0.13 to $0.84 \mu \mathrm{M}$. Apoptosis analysis revealed that compound $\mathbf{1 5}$ caused obvious induction of cell apoptosis. Compound $\mathbf{1 5}$ significantly down-regulated the expression level of the marker proteins (caspase-3 and PARP) in $\mathrm{H} 1299$ and $\mathrm{H} 1975$ cells, activated the transcription of IRE1 $\alpha$, increased the expression of GRP78 and XBP-1s, and finally induced apoptosis of H1299 cells. In vivo studies showed that 15 at a dose of $20 \mathrm{mg} / \mathrm{kg}$ suppressed tumor growth of $\mathrm{S} 180$ cell xenografts in icr mice significantly. Further molecular docking studies suggested that compound $\mathbf{1 5}$ could bind well with the ATPase domain of Topoisomerase-II. These data suggest that compound $\mathbf{1 5}$ is a promising agent for cancer therapy deserving further research.

Keywords: podophyllotoxin derivatives, biotin, anticancer activity, synthesis, apoptosis

\section{INTRODUCTION}

Cancer is a kind of frequently-occurring disease that seriously threatens human health. In recent years, more attention has been focused on targeting anti-cancer drugs. Development of targeted antitumor drugs, increase of bioavailability and decrease of toxicity are the key topics which are currently being studied. Research efforts in these topics have already led to the discovery of new drug leads and molecular scaffolds important for the development of novel antitumor agents 
(Fulda, 2010; Qiao et al., 2011; Wen et al., 2012). Currently, targeted cancer therapy has attracted a lot of interests in cancer research and has emerged as a new treatment option for various types of cancers.

Natural compounds are valuable sources with various structures, unique biological activities, and specific selectivity. Natural products have served as important sources of lead compounds for antitumor agents which have been developed for clinical use. However, many potential drugs lack tumor selectivity and often display significant toxic side effects, which hampers their development for clinical use (Holschneider et al., 1994; Bermejo et al., 2005). In order to enhance the therapeutic specificity of anticancer drugs, various targeting strategies have been explored, including antibodies (Wu and Ojima, 2004; Schrama et al., 2006; Lambert and Berkenblit, 2018), nanocarriers (Peer et al., 2007; Bonifácio et al., 2014; Hojjat-Farsangia et al., 2014; Senthilkumar et al., 2015), peptides (Mastrobattista et al., 1999; Dharap et al., 2005), and vitamins (Sawant et al., 2008; Chen et al., 2010; Guaragna et al., 2012). In each case, molecular features overexpressed on cancer cells are being targeted.

It has been widely recognized that all living cells depend on vitamins for survival and growth and obviously cancer cells must require higher amount of vitamins to meet the need of their rapid growth. Consequently, in order to sustain their rapid cell growth and enhanced proliferation, many cancer cells over-express receptors for certain vitamins. Therefore, vitamin receptors on the surface of these cells are important biomarkers for the delivery of tumor-targeted drugs (RussellJonesa et al., 2004; Leamon, 2008; Lu and Low, 2012). Biotin (vitamin $\mathrm{H}$ ) is a nutrient required for cell growth, and tumor cells need substantially higher amounts of biotin than normal cells due to their rapid growth (Russell-Jonesa et al., 2004). Recent studies have shown that many cancer cell lines express higher levels of biotin receptors (BRs) than normal cells, e.g., L1210FR (leukemia), Ov2008 (ovarian), Colo-26 (colon), P815 (mastocytoma), M109 (lung), RENCA (renal), and 4T1 (breast) cancer cell lines (Russell-Jonesa et al., 2004; Chen et al., 2010). Thus, BR has emerged as a useful biomarker for targeted delivery of anti-tumor agent, and biotin as a tumor-targeting module has been successfully employed for the construction of small molecule antitumor drug conjugates (Chen et al., 2008; Ojima, 2008; Ojima et al., 2012).

The natural lignan podophyllotoxin (PPT, 1, Figure 1) is isolated from Dysosma versipellis and shows cytotoxic activity against a variety of cancer cell lines by inhibiting microtubule assembly. However, PPT lacks tumor specificity and its high toxicity toward normal cells prevents its use in clinic for cancer treatment (Jardine, 1980; Desbene and Giorgi-Renault, 2002; Liu et al., 2007). The biological activity of PPT has led to extensive structural modification, resulting in several clinically useful compounds including etoposide (2, Figure 1), a semisynthetic glucosidic cyclic acetal of PPT. Etoposide exerts cytotoxic activity by inhibiting DNA topoisomerase II and the discovery of its novel mechanism of action led to further studies on the structure-activity relationship of PPT derivatives resulted from the structural modification at the C-4-position (Reddy et al., 2008; Zhang et al., 2014). Studies have shown that improvement in topoisomerase II inhibitory activity, water solubility, cytotoxic activity, drug resistance profile, and antitumor spectra of this class of compounds might be achieved through rational modification at C-4 position (Bromberg et al., 2003).

With the aim to improve the therapeutic efficacy and reduce the toxic side effects of podophyllotoxin in the treatment of cancer, we have designed a group of biotin-podophyllotoxin (Bio-PT) conjugates by covalently linking a biotin residue to podophyllotoxin. Such Bio-PT conjugates are anticipated to be taken up by cells through receptor-mediated endocytosis and selective delivery of these conjugates to cancer cells may be achieved due to a higher level of biotion receptors expressed on cancer cells. Here we report the synthesis of 14 biotinylated podophyllotoxin derivatives and their anticancer activity against various cancer cell lines. The compound with the highest anticancer activity was further studied to reveal the anticancer mechanisms and its antitumor effect was evaluated through in vivo studies as well.

\section{MATERIALS AND METHODS}

\section{General Information}

All cancer cells were obtained from a Shanghai cell bank in China. All reagents were commercially available and were used without further purification unless otherwise indicated. Podophyllotoxin was purchased from Shanghai Yuanye BioTechnology Co., Ltd (Shanghai, China). Anhydrous solvents were obtained by distillation from the indicated systems immediately prior to use: dichloromethane from calcium hydride and tetrahydrofuran from sodium. Uncorrected melting points were measured on a Beijing Taike XT-4. Electrospray ionization mass spectrometry (ESI-MS) data were acquired on API Qstar Pulsar instrument; High resolution electrospray ionization mass spectrometry (HRESI-MS) data were obtained on LCMS-ITTOF (Shimadzu, Kyoto, Japan); All NMR spectra were recorded with Bruker AV-400 or DRX-500 or Bruker AVANCE III-600 (Bruker BioSpin GmbH, Rheinstetten, Germany) instruments, with tetramethylsilane (TMS) as an internal standard: chemical shifts $(\delta)$ are given in ppm and coupling constants $(J)$ in $\mathrm{Hz}$. Column chromatography (CC) are carried out using silica gel (200-300 mesh; Qingdao Makall Group CO., LTD; Qingdao; China). All reactions were monitored by analytical thin-layer chromatography (TLC), which was visualized by ultraviolet light $(254 \mathrm{~nm})$ and/or $10 \%$ phosphomolybdic acid/EtOH.

\section{Synthesis}

\section{General Procedure for the Preparation of Biotinylated Podophyllotoxin Derivatives 13-26}

$\mathrm{N}, \mathrm{N}^{\prime}$-diisopropylcarbodiimide (DIC, $0.6 \mathrm{mmol}$ ) and 4dimethylaminopyridine (DMAP, $0.2 \mathrm{mmol}$ ) were added to a solution of biotin or 6-biotinylaminocaproic acid (0.2 $\mathrm{mmol})$, podophyllotoxin or its derivative $(0.2 \mathrm{mmol})$ in $\mathrm{N}$, $\mathrm{N}$-dimethylformamide (DMF, $2.5 \mathrm{~mL}$ ). The reaction mixture was stirred at room temperature for $24 \mathrm{~h}$ under $\mathrm{N}_{2}$. Solvents were removed under reduced pressure. The residue was purified by chromatography over silica gel $\left(\mathrm{CHCl}_{3} / \mathrm{CH}_{3} \mathrm{OH}=9: 1\right)$ to afford the desired product. 


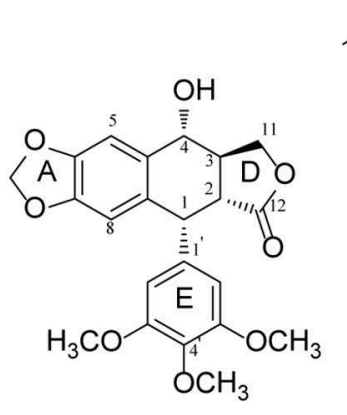

1 Podophyllotoxin

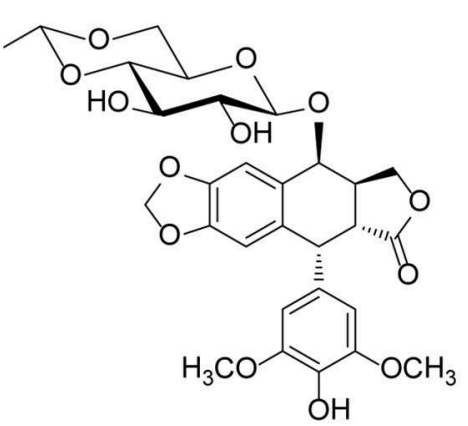

2 Etoposide<smiles>O=C(O)CCCCC1SC[C@H]2NC(=O)NC12</smiles><smiles>NC1CSC(CCCCC(=O)NCCCCCC(=O)O)C1NC(=O)O</smiles>

FIGURE 1 | Structure of Podophyllotoxin (1), Etoposide (2), Biotin (3), and 6-biotinylaminocaproic acid (4).

\section{4 $\alpha$-(biotin)-4-deoxypodophyllotoxin 13}

White amorphous powder, yield $64 \%$; m.p. $210-215^{\circ} \mathrm{C}$; $[\alpha]_{\mathrm{D}}^{26.2}$ : +34.6 ( c 0.13, DMSO); ${ }^{1} \mathrm{H}-\mathrm{NMR}\left(\mathrm{C}_{2} \mathrm{D}_{6} \mathrm{SO}, 500 \mathrm{MHz}\right) \delta 6.83$ (s, $\left.1 \mathrm{H}, \mathrm{C}^{5}-\mathrm{H}\right), 6.60\left(\mathrm{~s}, 1 \mathrm{H}, \mathrm{C}^{8}-\mathrm{H}\right), 6.52\left(\mathrm{~s}, 2 \mathrm{H}, \mathrm{C}^{2^{\prime}}, \mathrm{C}^{6^{\prime}}-\mathrm{H}\right), 6.01-5.98$ $\left(\mathrm{m}, 2 \mathrm{H}, \mathrm{OCH}_{2} \mathrm{O}\right), 5.67\left(\mathrm{~d}, 1 \mathrm{H}, J=6.0 \mathrm{~Hz}, \mathrm{C}^{4}-\mathrm{H}\right), 4.46-4.42(\mathrm{~m}$, $1 \mathrm{H}), 4.29\left(\mathrm{~d}, 1 \mathrm{H}, J=4.5 \mathrm{~Hz}, \mathrm{C}^{1}-\mathrm{H}\right), 4.27-4.23(\mathrm{~m}, 2 \mathrm{H}), 4.10-4.04$ $(\mathrm{m}, 1 \mathrm{H}), 3.70\left(\mathrm{~s}, 6 \mathrm{H}, \mathrm{C}^{3^{\prime}}, \mathrm{C}^{5^{\prime}}-\mathrm{OCH}_{3}\right), 3.62\left(\mathrm{~s}, 3 \mathrm{H}, \mathrm{C}^{4^{\prime}}-\mathrm{OCH}_{3}\right)$, $3.05-3.01(\mathrm{~m}, 1 \mathrm{H}), 2.79\left(\mathrm{dd}, 1 \mathrm{H}, J=4.5 \mathrm{~Hz}, 11.5 \mathrm{~Hz}, \mathrm{C}^{2}-\mathrm{H}\right), 2.57-$ $2.54(\mathrm{~m}, 2 \mathrm{H}), 2.43-2.40\left(\mathrm{~m}, 1 \mathrm{H}, \mathrm{C}^{3}-\mathrm{H}\right), 2.16(\mathrm{t}, 2 \mathrm{H}, J=9.0 \mathrm{~Hz}$, $\left.\mathrm{C}^{8^{\prime \prime \prime}}-\mathrm{CH}_{2}\right), 1.58-1.52(\mathrm{~m}, 2 \mathrm{H}), 1.42-4.40(\mathrm{~m}, 2 \mathrm{H}), 1.26-1.21(\mathrm{~m}$, $2 \mathrm{H}) ;{ }^{13} \mathrm{C}-\mathrm{NMR}\left(\mathrm{C}_{2} \mathrm{D}_{6} \mathrm{SO}, 100 \mathrm{MHz}\right) \delta 177.5(\mathrm{C}-12), 172.6$ (C$\left.7^{\prime \prime \prime}\right), 162.8$ (C-16'"'), 152.7 (C-3', C-5'), 147.5 (C-7), 146.3 (C-6), 138.4 (C-1'), 136.0 (C-4'), 132.4 (C-9), 126.8 (C-10), 109.2 (C-5), 108.2 (C-8), $105.6\left(\mathrm{C}-2^{\prime}, \mathrm{C}^{\prime} 6^{\prime}\right), 101.3\left(\mathrm{OCH}_{2} \mathrm{O}\right), 72.1(\mathrm{C}-11), 70.5$ (C-4), $61.0\left(\mathrm{C}-13^{\prime \prime \prime}\right), 60.0\left(\mathrm{C}-14^{\prime \prime \prime}\right), 59.2\left(4^{\prime}-\mathrm{OCH}_{3}\right), 55.9\left(3^{\prime}, 5^{\prime}-\right.$ $\left.\mathrm{OCH}_{3}\right), 55.3$ (C-12'"'), 43.6 (C-2), 43.5 (C-1), 40.1 (C-15'"'), 38.9 (C-3), 33.4 (C-8'"'), 28.0, 27.9, 24.4; ESIMS: m/z $641[\mathrm{M}+\mathrm{H}]$ ${ }^{+}$, HRESIMS: calcd for $\mathrm{C}_{32} \mathrm{H}_{36} \mathrm{~N}_{2} \mathrm{O}_{10} \mathrm{SH}[\mathrm{M}+\mathrm{H}]^{+}$641.2138, found 641.2163 .

\section{$4 \alpha$-(6-biotinylaminocaproic acid)-4-deoxypodophyllotoxin 14}

White amorphous powder, yield $54 \%$; m.p. $147-149^{\circ} \mathrm{C} ;[\alpha]_{\mathrm{D}}^{26.0}$ : -14.6 (c 0.10, Pyridine); ${ }^{1} \mathrm{H}-\mathrm{NMR}\left(\mathrm{CDCl}_{3}, 400 \mathrm{MHz}\right) \delta 6.72$ (s, $\left.1 \mathrm{H}, \mathrm{C}^{5}-\mathrm{H}\right), 6.50\left(\mathrm{~s}, 1 \mathrm{H}, \mathrm{C}^{8}-\mathrm{H}\right), 6.35\left(\mathrm{~s}, 2 \mathrm{H}, \mathrm{C}^{2^{\prime}}, \mathrm{C}^{6^{\prime}}-\mathrm{H}\right), 5.96$ $\left(\mathrm{d}, 2 \mathrm{H}, J=4.0 \mathrm{~Hz}, \mathrm{OCH}_{2} \mathrm{O}\right), 5.85\left(\mathrm{~d}, 1 \mathrm{H}, J=9.2 \mathrm{~Hz}, \mathrm{C}^{4}-\mathrm{H}\right)$, $4.57\left(\mathrm{~d}, 1 \mathrm{H}, J=4.0 \mathrm{~Hz}, \mathrm{C}^{1}-\mathrm{H}\right), 4.50-4.48(\mathrm{~m}, 1 \mathrm{H}), 4.34-4.27(\mathrm{~m}$, $2 \mathrm{H}), 4.17(\mathrm{t}, 1 \mathrm{H}, J=9.6 \mathrm{~Hz}), 3.77\left(\mathrm{~s}, 3 \mathrm{H}, \mathrm{C}^{4^{\prime}}-\mathrm{OCH}_{3}\right), 3.73(\mathrm{~s}$, $\left.6 \mathrm{H}, \mathrm{C}^{3^{\prime}}, \mathrm{C}^{5^{\prime}}-\mathrm{OCH}_{3}\right), 3.19-3.12\left(\mathrm{~m}, 2 \mathrm{H}, \mathrm{C}^{12^{\prime \prime \prime}}-\mathrm{H}, \mathrm{C}^{3}-\mathrm{H}\right), 2.94-$ $2.89\left(\mathrm{~m}, 1 \mathrm{H}, \mathrm{C}^{2}-\mathrm{H}\right), 2.88-2.84(\mathrm{~m}, 2 \mathrm{H}), 2.79-2.69(\mathrm{~m}, 2 \mathrm{H}), 2.40$ $\left(\mathrm{t}, 2 \mathrm{H}, J=7.2 \mathrm{~Hz}, \mathrm{C}^{2^{\prime \prime \prime}}-\mathrm{H}\right), 2.18\left(\mathrm{t}, 2 \mathrm{H}, J=6.8 \mathrm{~Hz}, \mathrm{C}^{8^{\prime \prime \prime}}-\mathrm{CH}_{2}\right)$, $1.68-1.64(\mathrm{~m}, 4 \mathrm{H}), 1.51-1.49(\mathrm{~m}, 2 \mathrm{H}), 1.40-1.35(\mathrm{~m}, 2 \mathrm{H}), 1.31-$ $1.29(\mathrm{~m}, 2 \mathrm{H}), 1.18-1.17(\mathrm{~m}, 2 \mathrm{H}) ;{ }^{13} \mathrm{C}-\mathrm{NMR}\left(\mathrm{CDCl}_{3}, 100 \mathrm{MHz}\right)$

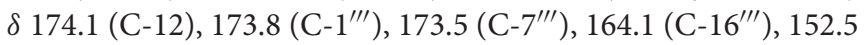
(C-3', C-5'), 148.1 (C-7), 147.5 (C-6), 136.9 (C-1'), 134.9 (C-4'), 132.2 (C-9), 128.3 (C-10), 109.7 (C-5), 108.0 (C-2', C-6'), 106.9 (C-8), $101.6\left(\mathrm{OCH}_{2} \mathrm{O}\right), 73.4(\mathrm{C}-11), 71.3(\mathrm{C}-4), 61.8\left(\mathrm{C}-13^{\prime \prime \prime}\right)$, $60.7\left(4^{\prime}-\mathrm{OCH}_{3}\right), 60.3\left(\mathrm{C}-14^{\prime \prime \prime}\right), 56.1\left(3^{\prime}, 5^{\prime}-\mathrm{OCH}_{3}\right), 55.7\left(\mathrm{C}-12^{\prime \prime \prime}\right)$, 45.4 (C-2), 43.6 (C-1), 40.5, 39.2, 38.7, 35.8, 34.1, 29.2, 28.2, 28.0,
26.4, 25.3, 24.5; ESIMS: $m / z 789\left[\mathrm{M}+\mathrm{Cl}^{-}{ }^{-}\right.$, HRESIMS: calcd for $\mathrm{C}_{38} \mathrm{H}_{47} \mathrm{~N}_{3} \mathrm{O}_{11} \mathrm{SCl}[\mathrm{M}+\mathrm{Cl}]^{-}$789.2625, found 789.2625.

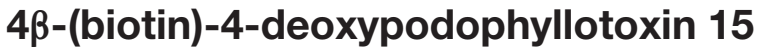

White amorphous powder, yield $65 \%$; m.p. $110-112^{\circ} \mathrm{C} ;[\alpha]_{\mathrm{D}}^{26.9}$ : $-51.6\left(c 0.21, \mathrm{CHCl}_{3}\right) ;{ }^{1} \mathrm{H}-\mathrm{NMR}\left(\mathrm{CDCl}_{3}, 400 \mathrm{MHz}\right) \delta 6.86(\mathrm{~s}, 1 \mathrm{H}$, $\left.\mathrm{C}^{5}-\mathrm{H}\right), 6.54\left(\mathrm{~s}, 1 \mathrm{H}, \mathrm{C}^{8}-\mathrm{H}\right), 6.26\left(\mathrm{~s}, 2 \mathrm{H}, \mathrm{C}^{2^{\prime}}, \mathrm{C}^{6^{\prime}}-\mathrm{H}\right), 6.14(\mathrm{~d}, 1 \mathrm{H}$, $\left.J=3.2 \mathrm{~Hz}, \mathrm{C}^{4}-\mathrm{H}\right), 5.98-5.96\left(\mathrm{~m}, 2 \mathrm{H}, \mathrm{OCH}_{2} \mathrm{O}\right), 4.66(\mathrm{~d}, 1 \mathrm{H}, J=$ $\left.4.8 \mathrm{~Hz}, \mathrm{C}^{1}-\mathrm{H}\right), 4.52-4.49(\mathrm{~m}, 1 \mathrm{H}), 4.36-4.28(\mathrm{~m}, 2 \mathrm{H}), 3.88(\mathrm{t}, 1 \mathrm{H}$, $J=8.8 \mathrm{~Hz}), 3.79\left(\mathrm{~s}, 6 \mathrm{H}, \mathrm{C}^{3^{\prime}}, \mathrm{C}^{5^{\prime}}-\mathrm{OCH}_{3}\right), 3.73\left(\mathrm{~s}, 3 \mathrm{H}, \mathrm{C}^{4^{\prime}}-\mathrm{OCH}_{3}\right)$, $3.24(\mathrm{dd}, 1 \mathrm{H}, J=5.2 \mathrm{~Hz}, 14.4 \mathrm{~Hz}), 3.15-3.10(\mathrm{~m}, 1 \mathrm{H}), 2.99-2.96$ (m, $1 \mathrm{H}), 2.89\left(\mathrm{dd}, 1 \mathrm{H}, J=4.8 \mathrm{~Hz}, 12.8 \mathrm{~Hz}, \mathrm{C}^{2}-\mathrm{H}\right), 2.75-2.71(\mathrm{~m}$, $2 \mathrm{H}), 2.38\left(\mathrm{t}, 2 \mathrm{H}, J=7.2 \mathrm{~Hz}, \mathrm{C}^{8^{\prime \prime \prime}}-\mathrm{CH}_{2}\right), 1.71-1.66(\mathrm{~m}, 4 \mathrm{H}), 1.45-$ $1.43(\mathrm{~m}, 2 \mathrm{H}) ;{ }^{13} \mathrm{C}-\mathrm{NMR}\left(\mathrm{CDCl}_{3}, 100 \mathrm{MHz}\right) \delta 174.2(\mathrm{C}-12), 173.3$

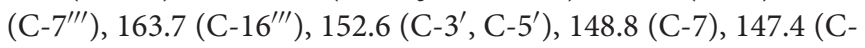
6), $137.2\left(\mathrm{C}-1^{\prime}\right), 134.6$ (C-4'), 132.9 (C-9), 127.8 (C-10), 110.2 (C5), 109.5 (C-8), $108.0\left(\mathrm{C}-2^{\prime}, \mathrm{C}-6^{\prime}\right), 101.7\left(\mathrm{OCH}_{2} \mathrm{O}\right), 68.0(\mathrm{C}-11)$, $67.5(\mathrm{C}-4), 62.1\left(\mathrm{C}-13^{\prime \prime \prime}\right), 60.7\left(4^{\prime}-\mathrm{OCH}_{3}\right), 60.3\left(\mathrm{C}-14^{\prime \prime \prime}\right), 56.2\left(3^{\prime}\right.$, $\left.5^{\prime}-\mathrm{OCH}_{3}\right), 55.4\left(\mathrm{C}-12^{\prime \prime \prime}\right), 43.8$ (C-2), 41.5 (C-1), 40.4, 36.7, 34.0, 28.4, 28.1, 24.7; ESIMS: $m / z 663[\mathrm{M}+\mathrm{Na}]^{+}$, HRESIMS: calcd for $\mathrm{C}_{32} \mathrm{H}_{36} \mathrm{~N}_{2} \mathrm{O}_{10} \mathrm{SNa}[\mathrm{M}+\mathrm{Na}]^{+} 663.1983$, found 663.1980 .

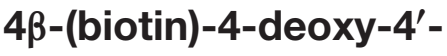 demethylpodophyllotoxin 16}

White amorphous powder, yield 59\%; m.p. $153-159^{\circ} \mathrm{C} ;[\alpha]_{\mathrm{D}}^{26.4}$ : -69.8 (c 0.17, Pyridine); ${ }^{1} \mathrm{H}-\mathrm{NMR}\left(\mathrm{C}_{5} \mathrm{D}_{5} \mathrm{~N}, 500 \mathrm{MHz}\right) \delta 7.64$ $\left(\mathrm{s}, 1 \mathrm{H}, \mathrm{C}^{5}-\mathrm{H}\right), 7.50\left(\mathrm{~s}, 1 \mathrm{H}, \mathrm{C}^{8}-\mathrm{H}\right), 7.24\left(\mathrm{~s}, 2 \mathrm{H}, \mathrm{C}^{2^{\prime}}, \mathrm{C}^{6^{\prime}}-\mathrm{H}\right), 6.82$ $\left(\mathrm{d}, 1 \mathrm{H}, J=3.2 \mathrm{~Hz}, \mathrm{C}^{4}-\mathrm{H}\right), 6.02-6.00\left(\mathrm{~m}, 2 \mathrm{H}, \mathrm{OCH}_{2} \mathrm{O}\right), 4.89(\mathrm{~d}$, $\left.1 \mathrm{H}, J=5.3 \mathrm{~Hz}, \mathrm{C}^{1}-\mathrm{H}\right), 4.69(\mathrm{t}, 1 \mathrm{H}, J=2.3 \mathrm{~Hz}), 4.54(\mathrm{t}, 1 \mathrm{H}, J=$ $2.1 \mathrm{~Hz}), 4.40-4.37(\mathrm{~m}, 1 \mathrm{H}), 3.85-3.84(\mathrm{~m}, 1 \mathrm{H}), 3.71\left(\mathrm{~s}, 6 \mathrm{H}, \mathrm{C}^{3^{\prime}}\right.$, $\left.\mathrm{C}^{5^{\prime}}-\mathrm{OCH}_{3}\right), 3.22-3.19(\mathrm{~m}, 2 \mathrm{H}), 3.92(\mathrm{dd}, 1 \mathrm{H}, J=5.0 \mathrm{~Hz}, 10.0 \mathrm{~Hz}$, $\left.\mathrm{C}^{2}-\mathrm{H}\right), 2.89-2.86(\mathrm{~m}, 2 \mathrm{H}), 2.63\left(\mathrm{t}, 2 \mathrm{H}, J=7.3 \mathrm{~Hz}, \mathrm{C}^{8^{\prime \prime \prime}}-\mathrm{CH}_{2}\right)$, $1.77-1.74(\mathrm{~m}, 2 \mathrm{H}), 1.61-1.57(\mathrm{~m}, 2 \mathrm{H}), 1.28-1.26(\mathrm{~m}, 2 \mathrm{H}) ;{ }^{13} \mathrm{C}-$ NMR $\left(\mathrm{C}_{5} \mathrm{D}_{5} \mathrm{~N}, 125 \mathrm{MHz}\right) \delta 175.7$ (C-12), $171.3\left(\mathrm{C}-7^{\prime \prime \prime}\right), 164.3$ (C-16'"'), 152.3 (C-3', C-5'), 148.4 (C-7), 147.7 (C-6), 139.5 (C$\left.1^{\prime}\right), 136.1\left(\mathrm{C}-4^{\prime}\right), 134.4(\mathrm{C}-9), 132.0$ (C-10), 110.6 (C-5), 110.4 (C-8), $108.5\left(\mathrm{C}-2^{\prime}, \mathrm{C}-6^{\prime}\right), 101.9\left(\mathrm{OCH}_{2} \mathrm{O}\right), 68.4(\mathrm{C}-11), 66.3(\mathrm{C}-$ 4), $62.5\left(\mathrm{C}-13^{\prime \prime \prime}\right), 60.6\left(\mathrm{C}-14^{\prime \prime \prime}\right), 56.3\left(3^{\prime}, 5^{\prime}-\mathrm{OCH}_{3}\right), 56.2\left(\mathrm{C}-12^{\prime \prime \prime}\right)$, 44.7 (C-2), 41.1 (C-1), 41.0, 39.7, 33.9, 29.1, 28.8, 25.4; ESIMS: 
$m / z 661[\mathrm{M}+\mathrm{Cl}]^{-}$, HRESIMS: calcd for $\mathrm{C}_{31} \mathrm{H}_{34} \mathrm{~N}_{2} \mathrm{O}_{10} \mathrm{SH}[\mathrm{M}+$ $\mathrm{H}]^{+}$627.2007, found 627.1978.

\section{$4 \beta$-(6-biotinylaminocaproic acid)-4-deoxypodophyllotoxin 17}

White amorphous powder, yield $57 \%$; m.p. $101-102^{\circ} \mathrm{C} ;[\alpha]_{\mathrm{D}}^{27.0}$ : -41.9 (c 0.13, $\left.\mathrm{CHCl}_{3}\right) ;{ }^{1} \mathrm{H}-\mathrm{NMR}\left(\mathrm{CDCl}_{3}, 400 \mathrm{MHz}\right) \delta 6.84(\mathrm{~s}$, $\left.1 \mathrm{H}, \mathrm{C}^{5}-\mathrm{H}\right), 6.54\left(\mathrm{~s}, 1 \mathrm{H}, \mathrm{C}^{8}-\mathrm{H}\right), 6.26\left(\mathrm{~s}, 2 \mathrm{H}, \mathrm{C}^{2^{\prime}}, \mathrm{C}^{6^{\prime}}-\mathrm{H}\right), 6.14(\mathrm{~d}$, $\left.2 \mathrm{H}, J=3.2 \mathrm{~Hz}, \mathrm{C}^{4}-\mathrm{H}\right), 5.99-5.98\left(\mathrm{~m}, 2 \mathrm{H}, \mathrm{OCH}_{2} \mathrm{O}\right), 4.66(\mathrm{~d}, 1 \mathrm{H}$, $\left.J=4.8 \mathrm{~Hz}, \mathrm{C}^{1}-\mathrm{H}\right), 4.52-4.50(\mathrm{~m}, 1 \mathrm{H}), 4.36-4.32(\mathrm{~m}, 2 \mathrm{H}), 3.87$ $(\mathrm{t}, 1 \mathrm{H}, J=8.8 \mathrm{~Hz}), 3.79\left(\mathrm{~s}, 6 \mathrm{H}, \mathrm{C}^{3^{\prime}}, \mathrm{C}^{5^{\prime}}-\mathrm{OCH}_{3}\right), 3.73(\mathrm{~s}, 3 \mathrm{H}$, $\left.\mathrm{C}^{4^{\prime}}-\mathrm{OCH}_{3}\right), 3.25-3.20(\mathrm{~m}, 2 \mathrm{H}), 3.16-3.10(\mathrm{~m}, 1 \mathrm{H}), 3.01-2.96(\mathrm{~m}$, $\left.1 \mathrm{H}, \mathrm{C}^{3}-\mathrm{H}\right), 2.89\left(\mathrm{dd}, 1 \mathrm{H}, J=4.8 \mathrm{~Hz}, 12.8 \mathrm{~Hz}, \mathrm{C}^{2}-\mathrm{H}\right), 2.76-2.73$ $(\mathrm{m}, 2 \mathrm{H}), 2.36\left(\mathrm{t}, 2 \mathrm{H}, J=8.0 \mathrm{~Hz}, \mathrm{C}^{2^{\prime \prime \prime}}-\mathrm{CH}_{2}\right), 2.25(\mathrm{t}, 2 \mathrm{H}, J=$ $\left.6.4 \mathrm{~Hz}, \mathrm{C}^{8^{\prime \prime \prime}}-\mathrm{CH}_{2}\right), 1.68-1.63(\mathrm{~m}, 6 \mathrm{H}), 1.53-1.50(\mathrm{~m}, 2 \mathrm{H}), 1.44-$ $1.41(\mathrm{~m}, 2 \mathrm{H}), 1.35-1.33(\mathrm{~m}, 2 \mathrm{H}) ;{ }^{13} \mathrm{C}-\mathrm{NMR}\left(\mathrm{CDCl}_{3}, 100 \mathrm{MHz}\right)$ $\delta 174.3$ (C-12), 173.7 (C-1'"'), 173.3 (C-7'"'), 163.9 (C-16'"'), 152.6 (C-3', C-5'), 148.9 (C-7), 147.4 (C-6), 137.2 (C-1'), 134.6 (C-4'), 132.8 (C-9), 127.8 (C-10), 110.2 (C-5), 109.5 (C-8), 108.0 (C-2', C-6'), $101.7\left(\mathrm{OCH}_{2} \mathrm{O}\right), 67.9(\mathrm{C}-11), 67.5(\mathrm{C}-4), 61.9\left(\mathrm{C}-13^{\prime \prime \prime}\right)$, $60.7\left(4^{\prime}-\mathrm{OCH}_{3}\right), 60.4\left(\mathrm{C}-14^{\prime \prime \prime}\right), 56.2\left(3^{\prime}, 5^{\prime}-\mathrm{OCH}_{3}\right), 55.6\left(\mathrm{C}-12^{\prime \prime \prime}\right)$, 43.7 (C-2), 41.5 (C-1), 40.5, 39.3, 36.7, 35.7, 34.1, 29.1, 28.0, 27.9, 26.4 $25.7,24.5$; ESIMS: $m / z 788\left[\mathrm{M}+\mathrm{Cl}^{-}\right.$, HRESIMS: calcd for $\mathrm{C}_{38} \mathrm{H}_{47} \mathrm{~N}_{3} \mathrm{O}_{11} \mathrm{SCl}[\mathrm{M}+\mathrm{Cl}]^{-}$788.2625, found 788.2627.

\section{$4 \beta$-(6-biotinylaminocaproic acid)-4-deoxy- $4^{\prime}$-demethylpodophyllotoxin 18}

White amorphous powder, yield $67 \%$; m.p. $117^{\circ} \mathrm{C} ;[\alpha]_{\mathrm{D}}^{25.9}:-6.4$ (c $\left.0.11, \mathrm{CHCl}_{3}\right) ;{ }^{1} \mathrm{H}-\mathrm{NMR}\left(\mathrm{CDCl}_{3}, 400 \mathrm{MHz}\right) \delta 6.88\left(\mathrm{~s}, 1 \mathrm{H}, \mathrm{C}^{5}\right.$ $\mathrm{H}), 6.50\left(\mathrm{~s}, 1 \mathrm{H}, \mathrm{C}^{8}-\mathrm{H}\right), 6.29\left(\mathrm{~s}, 2 \mathrm{H}, \mathrm{C}^{2^{\prime}}, \mathrm{C}^{6^{\prime}}-\mathrm{H}\right), 5.96-5.94(\mathrm{~m}$, $\left.2 \mathrm{H}, \mathrm{OCH}_{2} \mathrm{O}\right), 4.79\left(\mathrm{~d}, 1 \mathrm{H}, J=3.2 \mathrm{~Hz}, \mathrm{C}^{4}-\mathrm{H}\right), 4.60(\mathrm{~d}, 1 \mathrm{H}, J$ $\left.=5.2 \mathrm{~Hz}, \mathrm{C}^{1}-\mathrm{H}\right), 4.49-4.46(\mathrm{~m}, 1 \mathrm{H}), 4.36-4.32(\mathrm{~m}, 2 \mathrm{H}), 4.30-$ $4.27(\mathrm{~m}, 1 \mathrm{H}), 3.65\left(\mathrm{~s}, 6 \mathrm{H}, \mathrm{C}^{3^{\prime}}, \mathrm{C}^{5^{\prime}}-\mathrm{OCH}_{3}\right), 3.30-3.28(\mathrm{~m}, 1 \mathrm{H})$, $3.22-3.18(\mathrm{~m}, 2 \mathrm{H}), 3.14-3.10(\mathrm{~m}, 1 \mathrm{H}), 2.86(\mathrm{dd}, 1 \mathrm{H}, J=5.2 \mathrm{~Hz}$, $\left.12.8 \mathrm{~Hz}, \mathrm{C}^{2}-\mathrm{H}\right), 2.79-2.76(\mathrm{~m}, 1 \mathrm{H}), 2.72-2.69(\mathrm{~m}, 1 \mathrm{H}), 2.56(\mathrm{t}$, $\left.2 \mathrm{H}, J=6.8 \mathrm{~Hz}, \mathrm{C}^{2^{\prime \prime \prime}}-\mathrm{CH}_{2}\right), 2.35-2.32(\mathrm{~m}, 4 \mathrm{H}), 2.14(\mathrm{t}, 2 \mathrm{H}, J=$ $\left.6.8 \mathrm{~Hz}, \mathrm{C}^{8 \prime \prime \prime}-\mathrm{CH}_{2}\right), 1.75-1.70(\mathrm{~m}, 2 \mathrm{H}), 1.66-1.60(\mathrm{~m}, 2 \mathrm{H}), 1.53-$ $1.50(\mathrm{~m}, 2 \mathrm{H}), 1.43-1.36(\mathrm{~m}, 2 \mathrm{H}) ;{ }^{13} \mathrm{C}-\mathrm{NMR}\left(\mathrm{CDCl}_{3}, 150 \mathrm{MHz}\right)$ $\delta 175.8(\mathrm{C}-12), 173.8\left(\mathrm{C}-1^{\prime \prime \prime}\right), 171.9\left(\mathrm{C}-7^{\prime \prime \prime}\right), 163.9\left(\mathrm{C}-16^{\prime \prime \prime}\right), 151.5$ $\left(\mathrm{C}-3^{\prime}, \mathrm{C}^{-} 5^{\prime}\right), 148.4$ (C-7), 147.5 (C-6), 138.2 (C-9), 132.3 (C-10), $131.5\left(\mathrm{C}-4^{\prime}\right), 127.7\left(\mathrm{C}-1^{\prime}\right), 110.4(\mathrm{C}-5), 109.6$ (C-8), 107.7 (C-2', C-6'), $101.7\left(\mathrm{OCH}_{2} \mathrm{O}\right), 68.1(\mathrm{C}-11), 66.3(\mathrm{C}-4), 62.1\left(\mathrm{C}-13^{\prime \prime \prime}\right), 60.5$ (C-14'”), $56.3\left(3^{\prime}, 5^{\prime}-\mathrm{OCH}_{3}\right), 55.6\left(\mathrm{C}-12^{\prime \prime \prime}\right), 44.1(\mathrm{C}-2), 40.7$ (C-1), 40.5, 39.4, 38.6, 35.8, 33.7, 29.0, 28.3, 28.1, 26.2, 25.6, 24.6; ESIMS: $m / z 738[\mathrm{M}-\mathrm{H}]^{-}$, HRESIMS: calcd for $\mathrm{C}_{37} \mathrm{H}_{45} \mathrm{~N}_{3} \mathrm{O}_{11} \mathrm{SH}[\mathrm{M}+$ $\mathrm{H}]^{+} 740.2848$, found 740.2809 .

\section{4/-amino-(biotin)-4- \\ deoxypodophyllotoxin 19}

White amorphous powder, yield $62 \%$; m.p. $120^{\circ} \mathrm{C} ;[\alpha]_{D}^{26.3}$ : +35.3 (c 0.22 , pyridine); ${ }^{1} \mathrm{H}-\mathrm{NMR}\left(\mathrm{C}_{5} \mathrm{D}_{5} \mathrm{~N}, 500 \mathrm{MHz}\right) \delta 6.90$ (s, $\left.1 \mathrm{H}, \mathrm{C}^{5}-\mathrm{H}\right), 6.70\left(\mathrm{~s}, 2 \mathrm{H}, \mathrm{C}^{2^{\prime}}, \mathrm{C}^{6^{\prime}}-\mathrm{H}\right), 6.67\left(\mathrm{~s}, 1 \mathrm{H}, \mathrm{C}^{8}-\mathrm{H}\right), 5.96-5.93$ $\left(\mathrm{m}, 2 \mathrm{H}, \mathrm{OCH}_{2} \mathrm{O}\right), 4.84\left(\mathrm{~s}, 1 \mathrm{H}, \mathrm{C}^{4}-\mathrm{H}\right), 4.58(\mathrm{~d}, 1 \mathrm{H}, J=6.0 \mathrm{~Hz}$, $\left.\mathrm{C}^{1}-\mathrm{H}\right), 4.56-4.54(\mathrm{~m}, 2 \mathrm{H}), 4.37-4.34(\mathrm{~m}, 1 \mathrm{H}), 4.32-4.28(\mathrm{~m}, 1 \mathrm{H})$, $3.90\left(\mathrm{~s}, 3 \mathrm{H}, \mathrm{C}^{4^{\prime}}-\mathrm{OCH}_{3}\right), 3.69\left(\mathrm{~s}, 6 \mathrm{H}, \mathrm{C}^{3^{\prime}}, \mathrm{C}^{5^{\prime}}-\mathrm{OCH}_{3}\right), 3.39(\mathrm{t}$, $1 \mathrm{H}, J=9.0 \mathrm{~Hz}), 3.17-3.12\left(\mathrm{~m}, 1 \mathrm{H}, \mathrm{C}^{3}-\mathrm{H}\right), 2.91(\mathrm{dd}, 1 \mathrm{H}, J=$ $\left.6.0 \mathrm{~Hz}, 12.0 \mathrm{~Hz}, \mathrm{C}^{2}-\mathrm{H}\right), 2.86-2.83(\mathrm{~m}, 2 \mathrm{H}), 2.15(\mathrm{t}, 2 \mathrm{H}, J=9.0 \mathrm{~Hz}$, $\left.\mathrm{C}^{8^{\prime \prime \prime}}-\mathrm{CH}_{2}\right), 1.79-1.72(\mathrm{~m}, 2 \mathrm{H}), 1.46-1.42(\mathrm{~m}, 2 \mathrm{H}), 1.40-1.37(\mathrm{~m}$, $2 \mathrm{H}) ;{ }^{13} \mathrm{C}-\mathrm{NMR}\left(\mathrm{C}_{5} \mathrm{D}_{5} \mathrm{~N}, 125 \mathrm{MHz}\right) \delta 178.1(\mathrm{C}-12), 173.2(\mathrm{C}-$ $\left.7^{\prime \prime \prime}\right), 164.2$ (C-16'"'), 154.2 (C-3', C-5'), 148.0 (C-7), 146.5 (C-6), $141.3\left(\mathrm{C}-1^{\prime}\right), 136.4\left(\mathrm{C}-4^{\prime}\right), 129.5$ (C-9), 124.1 (C-10), 112.2 (C5), $107.2(\mathrm{C}-8), 107.2\left(\mathrm{C}-2^{\prime}, \mathrm{C}-6^{\prime}\right), 101.7\left(\mathrm{OCH}_{2} \mathrm{O}\right), 64.2(\mathrm{C}-11)$, $62.5(\mathrm{C}-4), 60.7\left(\mathrm{C}-13^{\prime \prime \prime}\right), 60.6\left(4^{\prime}-\mathrm{OCH}_{3}\right), 56.8\left(\mathrm{C}-14^{\prime \prime \prime}\right), 56.4\left(3^{\prime}\right.$, $\left.5^{\prime}-\mathrm{OCH}_{3}\right), 56.3\left(\mathrm{C}-12^{\prime \prime \prime}\right), 51.7$ (C-2), $47.2(\mathrm{C}-1), 42.9,41.1,34.1$, 29.0 (2), 25.3; ESIMS: $m / z 662[\mathrm{M}+\mathrm{Na}]^{+}$, HRESIMS: calcd for $\mathrm{C}_{32} \mathrm{H}_{37} \mathrm{~N}_{3} \mathrm{O}_{9} \mathrm{SNa}[\mathrm{M}+\mathrm{Na}]^{+}$662.2143, found 662.2145.

\section{4 $\beta$-amino-(biotin)-4-deoxy-4' - demethylpodophyllotoxin 20}

White amorphous powder, yield $41 \%$; m.p. $153^{\circ} \mathrm{C} ;[\alpha]_{\mathrm{D}}^{26.0}:-22.6$ (c 0.10, $\left.\mathrm{CHCl}_{3}\right) ;{ }^{1} \mathrm{H}-\mathrm{NMR}\left(\mathrm{CDCl}_{3}, 600 \mathrm{MHz}\right) \delta 6.82\left(\mathrm{~s}, 1 \mathrm{H}, \mathrm{C}^{5}\right.$ $\mathrm{H}), 6.51\left(\mathrm{~s}, 1 \mathrm{H}, \mathrm{C}^{8}-\mathrm{H}\right), 6.44\left(\mathrm{~s}, 2 \mathrm{H}, \mathrm{C}^{2^{\prime}}, \mathrm{C}^{6^{\prime}}-\mathrm{H}\right), 5.98-5.94(\mathrm{~m}$, $\left.2 \mathrm{H}, \mathrm{OCH}_{2} \mathrm{O}\right), 5.62\left(\mathrm{~s}, 1 \mathrm{H}, \mathrm{C}^{4}-\mathrm{H}\right), 4.57\left(\mathrm{~d}, 1 \mathrm{H}, J=4.2 \mathrm{~Hz}, \mathrm{C}^{1}-\mathrm{H}\right)$, $4.47-4.45(\mathrm{~m}, 1 \mathrm{H}), 4.41(\mathrm{t}, 1 \mathrm{H}, J=7.8 \mathrm{~Hz}), 4.28-4.26(\mathrm{~m}, 1 \mathrm{H})$, $4.16(\mathrm{t}, 1 \mathrm{H}, J=9.6 \mathrm{~Hz}), 3.77\left(\mathrm{~s}, 6 \mathrm{H}, \mathrm{C}^{3^{\prime}}, \mathrm{C}^{5^{\prime}}-\mathrm{OCH}_{3}\right), 3.14-3.10$ (m, $1 \mathrm{H}), 2.90-2.86(\mathrm{~m}, 2 \mathrm{H}), 2.75-2.70\left(\mathrm{~m}, 1 \mathrm{H}, \mathrm{C}^{3}-\mathrm{H}\right), 2.65-2.63$ (m, $1 \mathrm{H}), 2.29-2.20(\mathrm{~m}, 2 \mathrm{H}), 1.74-1.69(\mathrm{~m}, 2 \mathrm{H}), 1.56-1.50(\mathrm{~m}$, 2H), 1.43-1.38 (m, 2H); ${ }^{13} \mathrm{C}-\mathrm{NMR}\left(\mathrm{CDCl}_{3}, 150 \mathrm{MHz}\right) \delta 175.0(\mathrm{C}-$ 12), 174.2 (C-7"'), 164.5 (C-16'"'), 147.7 (C-7), 147.7 (C-6), 146.6 (C-3', C-5'), 134.7 (C-1'), 132.0 (C-4'), 131.6 (C-9), 130.7 (C10), 110.4 (C-5), 109.2 (C-2', C-6'), 106.6 (C-8), $101.7\left(\mathrm{OCH}_{2} \mathrm{O}\right)$, 71.8 (C-11), 69.7 (C-4), 61.9 (C-13'”'), 60.4 (C-14'"'), 57.1 (3', $\left.5^{\prime}-\mathrm{OCH}_{3}\right), 56.1$ (C-12'"'), 46.3 (C-2), 43.9 (C-1), 40.8 39.5, 36.0, 28.5, 28.4, 25.9; ESIMS: $m / z 660$ [M $+\mathrm{Cl}^{-}{ }^{-}$, HRESIMS: calcd for $\mathrm{C}_{31} \mathrm{H}_{35} \mathrm{~N}_{3} \mathrm{O}_{9} \mathrm{SH}[\mathrm{M}+\mathrm{H}]^{+}$626.2167, found 626.2145 .

\section{4/-amino-(6-biotinylaminocaproic acid)-4-deoxypodophyllotoxin 21}

White amorphous powder, yield $46 \%$; m.p. $100^{\circ} \mathrm{C} ;[\alpha]_{\mathrm{D}}^{27.1}:+58.3$ (c 0.12, $\left.\mathrm{CHCl}_{3}\right) ;{ }^{1} \mathrm{H}-\mathrm{NMR}\left(\mathrm{CDCl}_{3}, 400 \mathrm{MHz}\right) \delta 7.48(\mathrm{~s}, 1 \mathrm{H}, \mathrm{NH})$, $6.66\left(\mathrm{~s}, 1 \mathrm{H}, \mathrm{C}^{5}-\mathrm{H}\right), 6.46\left(\mathrm{~s}, 1 \mathrm{H}, \mathrm{C}^{8}-\mathrm{H}\right), 6.29\left(\mathrm{~s}, 2 \mathrm{H}, \mathrm{C}^{2^{\prime}}, \mathrm{C}^{6^{\prime}}-\right.$ $\mathrm{H}), 5.94-5.90\left(\mathrm{~m}, 2 \mathrm{H}, \mathrm{OCH}_{2} \mathrm{O}\right), 4.50\left(\mathrm{~s}, 1 \mathrm{H}, \mathrm{C}^{4}-\mathrm{H}\right), 4.34-4.32$ $(\mathrm{m}, 2 \mathrm{H}), 4.19-4.17(\mathrm{~m}, 2 \mathrm{H}), 4.09-4.05(\mathrm{~m}, 1 \mathrm{H}), 3.81\left(\mathrm{~s}, 3 \mathrm{H}, \mathrm{C}^{4^{\prime}}\right.$ $\left.\mathrm{OCH}_{3}\right), 3.76\left(\mathrm{~s}, 6 \mathrm{H}, \mathrm{C}^{3^{\prime}}, \mathrm{C}^{5^{\prime}}-\mathrm{OCH}_{3}\right), 3.19-3.13(\mathrm{~m}, 4 \mathrm{H}), 2.87-$ $2.85(\mathrm{~m}, 2 \mathrm{H}), 2.75-2.72(\mathrm{~m}, 1 \mathrm{H}), 2.52-2.50\left(\mathrm{~m}, 2 \mathrm{H}, \mathrm{C}^{2 \prime \prime \prime}-\mathrm{CH}_{2}\right)$, 2.24-2.23 (m, 6H), 1.69-1.64 (m, 2H), 1.51-1.48 (m, 2H), 1.45$1.42(\mathrm{~m}, 2 \mathrm{H}), 1.29-1.24(\mathrm{~m}, 2 \mathrm{H}) ;{ }^{13} \mathrm{C}-\mathrm{NMR}\left(\mathrm{CDCl}_{3}, 100 \mathrm{MHz}\right)$ $\delta 178.2(\mathrm{C}-12), 173.8\left(\mathrm{C}-1^{\prime \prime \prime}\right), 173.4\left(\mathrm{C}-7^{\prime \prime \prime}\right), 164.1\left(\mathrm{C}-16^{\prime \prime \prime}\right), 153.2$ (C-3', C-5'), 147.6 (C-7), 146.0 (C-6), $140.0\left(\mathrm{C}-1^{\prime}\right), 136.7$ (C-4'), 134.2 (C-9), 128.4 (C-10), 111.8 (C-5), 106.7 (C-8), 105.8 (C-2', C-6'), $101.2\left(\mathrm{OCH}_{2} \mathrm{O}\right), 63.4(\mathrm{C}-11), 61.9(\mathrm{C}-4), 60.8\left(4^{\prime}-\mathrm{OCH}_{3}\right)$, $60.4\left(\mathrm{C}-13^{\prime \prime \prime}\right), 56.6\left(\mathrm{C}-14^{\prime \prime \prime}\right), 56.2\left(3^{\prime}, 5^{\prime}-\mathrm{OCH}_{3}\right), 55.6\left(\mathrm{C}-12^{\prime \prime \prime}\right)$, 50.9 (C-2), 46.2 (C-1), 42.1, 40.5, 39.3, 35.6, 33.8, 28.9, 28.0, 27.9, 26.2, 25.7, 24.4; ESIMS: $m / z 775[\mathrm{M}+\mathrm{Na}]^{+}$, HRESIMS: calcd for $\mathrm{C}_{38} \mathrm{H}_{48} \mathrm{~N}_{4} \mathrm{O}_{10} \mathrm{SNa}[\mathrm{M}+\mathrm{Na}]^{+}$753.3164, found 753.3154.

\section{4 $\beta$-amino-(6-biotinylaminocaproic acid)-4- deoxy-4'-demethylpodophyllotoxin 22}

White amorphous powder, yield $64 \%$; m.p. $100^{\circ} \mathrm{C} ;[\alpha]_{\mathrm{D}}^{26.2}:+15.1$ (c 0.24, DMSO); ${ }^{1} \mathrm{H}-\mathrm{NMR}\left(\mathrm{C}_{2} \mathrm{D}_{6} \mathrm{SO}, 500 \mathrm{MHz}\right) \delta 8.28(\mathrm{~s}, 1 \mathrm{H}$, $\mathrm{NH}), 7.78(\mathrm{~s}, 1 \mathrm{H}, \mathrm{NH}), 6.83\left(\mathrm{~s}, 1 \mathrm{H}, \mathrm{C}^{5}-\mathrm{H}\right), 6.45\left(\mathrm{~s}, 1 \mathrm{H}, \mathrm{C}^{8}-\mathrm{H}\right), 6.38$ $\left(\mathrm{s}, 2 \mathrm{H}, \mathrm{C}^{2^{\prime}}, \mathrm{C}^{6^{\prime}}-\mathrm{H}\right), 5.97-5.92\left(\mathrm{~m}, 2 \mathrm{H}, \mathrm{OCH}_{2} \mathrm{O}\right), 4.28(\mathrm{~d}, 1 \mathrm{H}, \mathrm{J}=$ 
$\left.5.0 \mathrm{~Hz}, \mathrm{C}^{4}-\mathrm{H}\right), 4.26\left(\mathrm{~d}, 1 \mathrm{H}, J=5.0 \mathrm{~Hz}, \mathrm{C}^{1}-\mathrm{H}\right), 4.24(\mathrm{~s}, 1 \mathrm{H}), 4.16$ $(\mathrm{s}, 1 \mathrm{H}), 4.11-4.09(\mathrm{~m}, 2 \mathrm{H}), 3.64\left(\mathrm{~s}, 6 \mathrm{H}, \mathrm{C}^{3^{\prime}}, \mathrm{C}^{5^{\prime}}-\mathrm{OCH}_{3}\right), 3.30-3.27$ (m, 2H), 3.09-3.05 (m, 1H, C $3-\mathrm{H}), 3.02-2.99(\mathrm{~m}, 2 \mathrm{H}), 2.78(\mathrm{dd}$, $\left.1 \mathrm{H}, J=5.0 \mathrm{~Hz}, 12.0 \mathrm{~Hz}, \mathrm{C}^{2}-\mathrm{H}\right), 2.54-2.50(\mathrm{~m}, 3 \mathrm{H}), 2.02(\mathrm{t}, 2 \mathrm{H}, J$ $\left.=7.5 \mathrm{~Hz}, \mathrm{C}^{8^{\prime \prime \prime}}-\mathrm{CH}_{2}\right), 1.61-1.58(\mathrm{~m}, 4 \mathrm{H}), 1.50-1.44(\mathrm{~m}, 2 \mathrm{H}), 1.42-$ $1.38(\mathrm{~m}, 2 \mathrm{H}), 1.35-1.30(\mathrm{~m}, 2 \mathrm{H}), 1.27-1.24(\mathrm{~m}, 2 \mathrm{H}) ;{ }^{13} \mathrm{C}-\mathrm{NMR}$ $\left(\mathrm{C}_{2} \mathrm{D}_{6} \mathrm{SO}, 125 \mathrm{MHz}\right) \delta 177.2(\mathrm{C}-12), 171.9\left(\mathrm{C}-1^{\prime \prime \prime}\right), 170.8\left(\mathrm{C}-7^{\prime \prime \prime}\right)$, $162.7\left(\mathrm{C}-16^{\prime \prime \prime}\right), 151.4\left(\mathrm{C}-3^{\prime}, \mathrm{C}-5^{\prime}\right), 146.6$ (C-7), 145.2 (C-6), 143.4 $\left(\mathrm{C}-1^{\prime}\right), 136.1$ (C-4'), 128.4 (C-9), 126.7 (C-10), 111.1 (C-5), 106.9 (C-8), $105.4\left(\mathrm{C}-2^{\prime}, \mathrm{C}^{\prime} 6^{\prime}\right), 100.9\left(\mathrm{OCH}_{2} \mathrm{O}\right), 61.1(\mathrm{C}-4), 60.6(\mathrm{C}-11)$, $59.2\left(\mathrm{C}-13^{\prime \prime \prime}\right), 55.9\left(3^{\prime}, 5^{\prime}-\mathrm{OCH}_{3}\right), 55.4\left(\mathrm{C}-14^{\prime \prime \prime}\right), 54.9\left(\mathrm{C}-12^{\prime \prime \prime}\right)$, 50.1 (C-2), 45.6 (C-1), 44.8, 40.0, 38.2, 35.2, 33.0, 28.8, 28.2, 28.0, 25.6, 25.3, 24.3; ESIMS: $m / z 761[\mathrm{M}+\mathrm{Na}]^{+}$, HRESIMS: calcd for $\mathrm{C}_{37} \mathrm{H}_{46} \mathrm{~N}_{4} \mathrm{O}_{10} \mathrm{SH}[\mathrm{M}+\mathrm{Na}]^{+}$761.2827, found 761.2829.

\section{$4 \beta-\{[4-h y d r o x y m e t h y l-(b i o t i n)-1,2,3-$ triazol- 1-yl]\}-4-deoxypodophyllotoxin 23}

White amorphous powder, yield $39 \%$; m.p. $110^{\circ} \mathrm{C}$; $[\alpha]_{\mathrm{D}}^{27.1}$ : -21.6 (c 0.16, $\left.\mathrm{CHCl}_{3}\right) ;{ }^{1} \mathrm{H}-\mathrm{NMR}\left(\mathrm{CDCl}_{3}, 400 \mathrm{MHz}\right) \delta 7.39\left(\mathrm{~s}, 1 \mathrm{H}, \mathrm{C}^{5^{\prime \prime}}-\right.$ $\mathrm{H}), 6.65\left(\mathrm{~s}, 1 \mathrm{H}, \mathrm{C}^{5}-\mathrm{H}\right), 6.62\left(\mathrm{~s}, 1 \mathrm{H}, \mathrm{C}^{8}-\mathrm{H}\right), 6.32\left(\mathrm{~s}, 2 \mathrm{H}, \mathrm{C}^{2}, \mathrm{C}^{6^{\prime}}-\mathrm{H}\right)$, 6.10-6.00 (m, 3H, OCH $\left.{ }_{2} \mathrm{O}, \mathrm{C}^{4}-\mathrm{H}\right), 5.21-5.13\left(\mathrm{~m}, 2 \mathrm{H}, \mathrm{C}^{6^{\prime \prime}}-\mathrm{CH}_{2}\right)$, $4.76\left(\mathrm{~d}, 1 \mathrm{H}, J=4.0 \mathrm{~Hz}, \mathrm{C}^{1}-\mathrm{H}\right), 4.52-4.51(\mathrm{~m}, 1 \mathrm{H}), 4.40-4.26(\mathrm{~m}$, $3 \mathrm{H}), 3.80\left(\mathrm{~s}, 3 \mathrm{H}, \mathrm{C}^{4^{\prime}}-\mathrm{OCH}_{3}\right), 3.76\left(\mathrm{~s}, 6 \mathrm{H}, \mathrm{C}^{3^{\prime}}, \mathrm{C}^{5^{\prime}}-\mathrm{OCH}_{3}\right), 3.22-$ $3.13(\mathrm{~m}, 3 \mathrm{H}), 2.92-2.89(\mathrm{~m}, 1 \mathrm{H}), 2.77-2.73(\mathrm{~m}, 1 \mathrm{H}), 2.36(\mathrm{t}, 2 \mathrm{H}, J$ $\left.=6.0 \mathrm{~Hz}, \mathrm{C}^{8^{\prime \prime \prime}}-\mathrm{CH}_{2}\right), 1.86-1.83(\mathrm{~m}, 2 \mathrm{H}), 1.66-1.63(\mathrm{~m}, 2 \mathrm{H}), 1.42-$ $1.40(\mathrm{~m}, 2 \mathrm{H}) ;{ }^{13} \mathrm{C}-\mathrm{NMR}\left(\mathrm{CDCl}_{3}, 100 \mathrm{MHz}\right) \delta 173.6(\mathrm{C}-12), 173.6$ (C-7'"'), 152.7 (C-3', C-5'), 149.4 (C-7), 148.0 (C-6), 142.9 (C-4") $137.4\left(\mathrm{C}-1^{\prime}\right), 134.3\left(\mathrm{C}-4^{\prime}\right), 133.2$ (C-9), 124.5 (C-10), 124.3 (C$\left.5^{\prime \prime}\right), 110.5$ (C-5), $108.8(\mathrm{C}-8), 108.1\left(\mathrm{C}-2^{\prime}, \mathrm{C}^{\prime} 6^{\prime}\right), 102.0\left(\mathrm{OCH}_{2} \mathrm{O}\right)$, $67.4(\mathrm{C}-11), 62.0(\mathrm{C}-4), 60.7\left(4^{\prime}-\mathrm{OCH}_{3}\right), 60.3\left(\mathrm{C}-13^{\prime \prime \prime}\right), 58.7(\mathrm{C}-$ $\left.14^{\prime \prime \prime}\right), 57.3\left(\mathrm{C}^{6^{\prime \prime}}-\mathrm{CH}_{2}\right), 56.3\left(3^{\prime}, 5^{\prime}-\mathrm{OCH}_{3}\right), 55.4\left(\mathrm{C}-12^{\prime \prime \prime}\right), 43.6$ (C-2), 41.5 (C-1), 40.4 37.1, 33.6, 28.1, 25.6, 24.6; ESIMS: $m / z 744$ $[\mathrm{M}+\mathrm{Na}]^{+}$, HRESIMS: calcd for $\mathrm{C}_{35} \mathrm{H}_{39} \mathrm{~N}_{5} \mathrm{O}_{10} \mathrm{SNa}[\mathrm{M}+\mathrm{Na}]^{+}$ 744.2310 , found 744.2356 .

\section{$4 \beta-\{[4-h y d r o x y m e t h y l-$ (6-biotinylaminocaproic acid)-1,2,3-triazol- 1-yl]\}-4-deoxy-4' - demethylpodophyllotoxin 24}

White amorphous powder, yield $60 \%$; m.p. $104^{\circ} \mathrm{C}$; $[\alpha]_{\mathrm{D}}^{26.3}$ : -60.3 (c 0.19, Pyridine); ${ }^{1} \mathrm{H}-\mathrm{NMR}\left(\mathrm{CDCl}_{3}, 400 \mathrm{MHz}\right) \delta 7.74\left(\mathrm{~s}, 1 \mathrm{H}, \mathrm{C}^{5^{\prime \prime}}\right.$ $\mathrm{H}), 6.57\left(\mathrm{~s}, 1 \mathrm{H}, \mathrm{C}^{5}-\mathrm{H}\right), 6.45\left(\mathrm{~s}, 2 \mathrm{H}, \mathrm{C}^{2^{\prime}}, \mathrm{C}^{6^{\prime}}-\mathrm{H}\right), 6.20\left(\mathrm{~s}, 1 \mathrm{H}, \mathrm{C}^{8}-\mathrm{H}\right)$, 5.93-5.84 (m, 3H, $\left.\mathrm{OCH}_{2} \mathrm{O}, \mathrm{C}^{4}-\mathrm{H}\right), 5.23-5.15\left(\mathrm{~m}, 2 \mathrm{H}, \mathrm{C}^{6 \prime}-\mathrm{CH}_{2}\right)$, $4.65\left(\mathrm{~d}, 1 \mathrm{H}, J=4.0 \mathrm{~Hz}, \mathrm{C}^{1}-\mathrm{H}\right), 4.46-4.44(\mathrm{~m}, 1 \mathrm{H}), 4.22-4.16$ $(\mathrm{m}, 2 \mathrm{H}), 4.11(\mathrm{t}, 1 \mathrm{H}, J=9.2 \mathrm{~Hz}), 3.79\left(\mathrm{~s}, 6 \mathrm{H}, \mathrm{C}^{3^{\prime}}, \mathrm{C}^{5^{\prime}}-\mathrm{OCH}_{3}\right)$, $3.30-3.25(\mathrm{~m}, 1 \mathrm{H}), 3.03-2.99(\mathrm{~m}, 3 \mathrm{H}), 2.79(\mathrm{dd}, 1 \mathrm{H}, J=4.0 \mathrm{~Hz}$, $\left.10.0 \mathrm{~Hz}, \mathrm{C}^{2}-\mathrm{H}\right), 2.32\left(\mathrm{t}, 2 \mathrm{H}, J=6.8 \mathrm{~Hz}, \mathrm{C}^{8^{\prime \prime \prime}}-\mathrm{CH}_{2}\right), 1.65-1.55(\mathrm{~m}$, $4 \mathrm{H}), 1.35-1.33(\mathrm{~m}, 2 \mathrm{H}) ;{ }^{13} \mathrm{C}-\mathrm{NMR}\left(\mathrm{CDCl}_{3}, 100 \mathrm{MHz}\right) \delta 173.6$ (C-12), 173.5 (C-7'"'), 148.5 (C-7), 147.9 (C-6), 146.8 (C-3', C$\left.{ }^{5 \prime}\right), 143.4\left(\mathrm{C}-4^{\prime \prime}\right), 134.1\left(\mathrm{C}-1^{\prime}\right), 132.5\left(\mathrm{C}-4^{\prime}\right), 129.9$ (C-9), 126.6

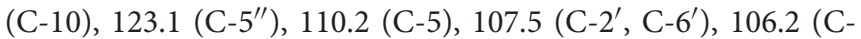
8), $101.8\left(\mathrm{OCH}_{2} \mathrm{O}\right), 70.1$ (C-11), $63.1(\mathrm{C}-4), 62.1\left(\mathrm{C}-13^{\prime \prime \prime}\right), 60.2$ $\left(\mathrm{C}-14^{\prime \prime \prime}\right), 57.3\left(\mathrm{C}^{6^{\prime \prime}}-\mathrm{CH}_{2}\right), 56.3\left(3^{\prime}, 5^{\prime}-\mathrm{OCH}_{3}\right), 55.4\left(\mathrm{C}-12^{\prime \prime \prime}\right), 45.8$ (C-2), 43.6 (C-1), 40.2 38.7, 33.5, 28.2, 28.1, 24.5; ESIMS: $m / z 730$ $[\mathrm{M}+\mathrm{Na}]^{+}$, HRESIMS: calcd for $\mathrm{C}_{34} \mathrm{H}_{37} \mathrm{~N}_{5} \mathrm{O}_{10} \mathrm{SH}[\mathrm{M}+\mathrm{H}]^{+}$ 708.2334, found 708.2302.

\section{$4 \beta-\{[4-h y d r o x y m e t h y l-$} (6-biotinylaminocaproic acid)-1,2,3-triazol1-yl]\}-4-deoxypodophyllotoxin 25

White amorphous powder, yield $47 \%$; m.p. $101^{\circ} \mathrm{C} ;[\alpha]_{\mathrm{D}}^{27.2}:-25.4$ (c 0.24, $\left.\mathrm{CHCl}_{3}\right) ;{ }^{1} \mathrm{H}-\mathrm{NMR}\left(\mathrm{CDCl}_{3}, 400 \mathrm{MHz}\right) \delta 7.41\left(\mathrm{~s}, 1 \mathrm{H}, \mathrm{C}^{5^{\prime \prime}}\right.$ $\mathrm{H}), 6.62\left(\mathrm{~s}, 1 \mathrm{H}, \mathrm{C}^{5}-\mathrm{H}\right), 6.60\left(\mathrm{~s}, 1 \mathrm{H}, \mathrm{C}^{8}-\mathrm{H}\right), 6.30\left(\mathrm{~s}, 2 \mathrm{H}, \mathrm{C}^{2^{\prime}}, \mathrm{C}^{6^{\prime}}-\mathrm{H}\right)$, $6.11\left(\mathrm{~d}, 1 \mathrm{H}, J=3.2 \mathrm{~Hz}, \mathrm{C}^{4}-\mathrm{H}\right), 6.00\left(\mathrm{~d}, 2 \mathrm{H}, J=4.8 \mathrm{~Hz}, \mathrm{OCH}_{2} \mathrm{O}\right)$, $5.15\left(\mathrm{~s}, 2 \mathrm{H}, \mathrm{C}^{6^{\prime \prime}}-\mathrm{CH}_{2}\right), 4.75\left(\mathrm{~d}, 1 \mathrm{H}, J=4.8 \mathrm{~Hz}, \mathrm{C}^{1}-\mathrm{H}\right), 4.52-4.49$ (m, $1 \mathrm{H}), 4.38-4.31(\mathrm{~m}, 3 \mathrm{H}), 3.79\left(\mathrm{~s}, 3 \mathrm{H}, \mathrm{C}^{4^{\prime}}-\mathrm{OCH}_{3}\right), 3.75(\mathrm{~s}, 6 \mathrm{H}$, $\left.\mathrm{C}^{3^{\prime}}, \mathrm{C}^{5^{\prime}}-\mathrm{OCH}_{3}\right), 3.20-3.13(\mathrm{~m}, 5 \mathrm{H}), 2.89-2.86(\mathrm{~m}, 1 \mathrm{H}), 2.74-2.71$ $(\mathrm{m}, 1 \mathrm{H}), 2.32\left(\mathrm{t}, 2 \mathrm{H}, J=7.2 \mathrm{~Hz}, \mathrm{C}^{2^{\prime \prime \prime}}-\mathrm{CH}_{2}\right), 2.22(\mathrm{t}, 2 \mathrm{H}, J=$ $\left.6.4 \mathrm{~Hz}, \mathrm{C}^{8^{\prime \prime \prime}}-\mathrm{CH}_{2}\right), 1.66-1.58(\mathrm{~m}, 6 \mathrm{H}), 1.49-1.46(\mathrm{~m}, 2 \mathrm{H}), 1.42-$ $1.40(\mathrm{~m}, 2 \mathrm{H}), 1.30-1.28(\mathrm{~m}, 2 \mathrm{H}) ;{ }^{13} \mathrm{C}-\mathrm{NMR}\left(\mathrm{CDCl}_{3}, 100 \mathrm{MHz}\right)$ $\delta 173.7(\mathrm{C}-12), 173.5\left(\mathrm{C}-1^{\prime \prime \prime}\right), 173.3\left(\mathrm{C}-7^{\prime \prime \prime}\right), 164.0\left(\mathrm{C}-16^{\prime \prime \prime}\right), 152.7$ (C-3', C-5'), 149.4 (C-7), 148.0 (C-6), 142.9 (C-4"'), $137.4\left(\mathrm{C}-1^{\prime}\right)$, $134.3\left(\mathrm{C}-4^{\prime}\right), 133.2$ (C-9), 124.4 (C-10), $124.3\left(\mathrm{C}-5^{\prime \prime}\right), 110.5$ (C-5), 108.8 (C-8), $108.1\left(\mathrm{C}-^{\prime}, \mathrm{C}^{\prime} \mathrm{6}^{\prime}\right), 102.0\left(\mathrm{OCH}_{2} \mathrm{O}\right), 67.4(\mathrm{C}-11), 61.8$ (C-4), $60.7\left(4^{\prime}-\mathrm{OCH}_{3}\right), 60.3\left(\mathrm{C}-13^{\prime \prime \prime}\right), 58.7\left(\mathrm{C}-14^{\prime \prime \prime}\right), 57.2\left(\mathrm{C}^{6^{\prime \prime}}\right.$ $\left.\mathrm{CH}_{2}\right), 56.3\left(3^{\prime}, 5^{\prime}-\mathrm{OCH}_{3}\right), 55.6\left(\mathrm{C}-12^{\prime \prime \prime}\right), 43.6(\mathrm{C}-2), 41.5(\mathrm{C}-1)$, 40.5 39.2, 37.1, 35.7, 33.8, 28.9, 28.1, 27.9, 26.1, 25.7, 24.3; ESIMS: $\mathrm{m} / z 857[\mathrm{M}+\mathrm{Na}]^{+}$, HRESIMS: calcd for $\mathrm{C}_{41} \mathrm{H}_{50} \mathrm{~N}_{6} \mathrm{O}_{11} \mathrm{SH}[\mathrm{M}+$ $\mathrm{H}]^{+}$835.3331, found 835.3338 .

\section{$4 \beta-\{[4-h y d r o x y m e t h y l-(6-$ biotinylaminocaproic acid)-1,2,3-triazol-1-yl]\}-4-deoxy-4' - demethylpodophyllotoxin 26}

White amorphous powder, yield $58 \%$; m.p. $119^{\circ} \mathrm{C} ;[\alpha]_{\mathrm{D}}^{27.1}:-9.6(c$ $\left.0.16, \mathrm{CHCl}_{3}\right) ;{ }^{1} \mathrm{H}-\mathrm{NMR}\left(\mathrm{CDCl}_{3}, 400 \mathrm{MHz}\right) \delta 7.36\left(\mathrm{~s}, 1 \mathrm{H}, \mathrm{C}^{5^{\prime \prime}}-\mathrm{H}\right)$, $6.60\left(\mathrm{~s}, 1 \mathrm{H}, \mathrm{C}^{5}-\mathrm{H}\right), 6.59\left(\mathrm{~s}, 1 \mathrm{H}, \mathrm{C}^{8}-\mathrm{H}\right), 6.33\left(\mathrm{~s}, 2 \mathrm{H}, \mathrm{C}^{2}, \mathrm{C}^{6^{\prime}}-\mathrm{H}\right)$, $6.10\left(\mathrm{~d}, 1 \mathrm{H}, J=4.0 \mathrm{~Hz}, \mathrm{C}^{4}-\mathrm{H}\right), 5.99-5.96\left(\mathrm{~m}, 2 \mathrm{H}, \mathrm{OCH}_{2} \mathrm{O}\right), 4.75$ $\left(\mathrm{s}, 1 \mathrm{H}, \mathrm{C}^{1}-\mathrm{H}\right), 4.68\left(\mathrm{~s}, 2 \mathrm{H}, \mathrm{C}^{6^{\prime \prime}}-\mathrm{CH}_{2}\right), 4.49-4.46(\mathrm{~m}, 1 \mathrm{H}), 4.37-$ $4.36(\mathrm{~m}, 1 \mathrm{H}), 4.30-4.27(\mathrm{~m}, 2 \mathrm{H}), 3.67\left(\mathrm{~s}, 6 \mathrm{H}, \mathrm{C}^{3^{\prime}}, \mathrm{C}^{5^{\prime}}-\mathrm{OCH}_{3}\right)$, $3.20-3.10(\mathrm{~m}, 6 \mathrm{H}), 2.85\left(\mathrm{dd}, 1 \mathrm{H}, J=4.8 \mathrm{~Hz}, 12.2 \mathrm{~Hz}, \mathrm{C}^{2}-\mathrm{H}\right), 2.64$ $\left(\mathrm{m}, 2 \mathrm{H}, J=7.2 \mathrm{~Hz}, \mathrm{C}^{2^{\prime \prime \prime}}-\mathrm{CH}_{2}\right), 2.56\left(\mathrm{t}, 2 \mathrm{H}, J=6.8 \mathrm{~Hz}, \mathrm{C}^{8^{\prime \prime \prime}}-\mathrm{CH}_{2}\right)$, 1.73-1.68 (m, 2H), 1.66-1.58 (m, 4H), 1.53-1.50 (m, 2H), 1.45$1.35(\mathrm{~m}, 4 \mathrm{H}) ;{ }^{13} \mathrm{C}-\mathrm{NMR}\left(\mathrm{CDCl}_{3}, 125 \mathrm{MHz}\right) \delta 174.0(\mathrm{C}-12), 173.6$ (C-1"'), 171.7 (C-7'"'), 164.0 (C-16'"'), 151.6 (C-3', C-5'), 149.4 (C-7), 148.1 (C-6), $148.0\left(\mathrm{C}-4^{\prime \prime}\right), 137.2\left(\mathrm{C}-1^{\prime}\right), 132.8$ (C-4'), 128.0 (C-9), 124.7 (C-10), 122.9 (C-5"), 110.5 (C-5), 108.9 (C-8), 107.6 $\left(\mathrm{C}-2^{\prime}, \mathrm{C}^{\prime} 6^{\prime}\right), 102.0\left(\mathrm{OCH}_{2} \mathrm{O}\right), 67.5(\mathrm{C}-11), 62.1(\mathrm{C}-4), 60.4(\mathrm{C}-$ $\left.13^{\prime \prime \prime}\right), 58.7\left(\mathrm{C}-14^{\prime \prime \prime}\right), 56.1\left(3^{\prime}, 5^{\prime}-\mathrm{OCH}_{3}\right), 55.6\left(\mathrm{C}^{6^{\prime \prime}}-\mathrm{CH}_{2}\right), 55.4(\mathrm{C}-$ $\left.12^{\prime \prime \prime}\right), 43.6$ (C-2), 41.4 (C-1), 40.1, 39.3, 37.0, 35.4, 33.5, 28.7, 28.1, 27.8, 26.0, 25.5, 24.4; ESIMS: $m / z 843[\mathrm{M}+\mathrm{Na}]^{+}$, HRESIMS: calcd for $\mathrm{C}_{40} \mathrm{H}_{48} \mathrm{~N}_{6} \mathrm{O}_{11} \mathrm{SH}[\mathrm{M}+\mathrm{H}]^{+}$821.3175, found 821.3195.

\section{Biology Assay Cell Culture}

All cell lines used in this study were cultured in DMEM or RMPI1640 medium (Hyclone, Logan, UT, USA) which is supplemented with $50 \mathrm{mg} / \mathrm{L}$ of streptomycin, $50 \mathrm{IU} / \mathrm{ml}$ of penicillin (Solarbio, Beijing, China) and $10 \%$ fetal bovine serum (HyClone, CA, USA) in a humidified $5 \% \mathrm{CO}_{2}$ incubator at $37^{\circ} \mathrm{C}$. All cells were sub-cultured 3 times/week by trypsinisation. 
<smiles>COc1cc([C@H]2c3cc4c(cc3[C@@H](O)[C@@H]3COC(=O)[C@H]23)OCO4)cc(OC)c1OC</smiles>

1<smiles>[3H][3H]</smiles><smiles>[R]Oc1c(OC)cc([C@H]2c3cc4c(cc3[C@@H](O)[C@@H]3COC(=O)[C@H]23)OCO4)cc1OC</smiles>

$5 \mathrm{R}=\mathrm{CH}_{3}$ $6 \mathrm{R}=\mathrm{H}$<smiles>COc1cc([C@H]2c3cc4c(cc3C(N)C3COC(=O)[C@@H]32)OCO4)cc(OC)c1O</smiles>

$9 \mathrm{R}=\mathrm{CH}_{3}$

$10 \mathrm{R}=\mathrm{H}$<smiles>[R2]Oc1c(OC)cc([C@H]2c3cc4c(cc3[C@@H](NC(C)C)[C@@H]3COC(=O)[C@H]23)OCO4)cc1OC</smiles>

$7 \mathrm{R}=\mathrm{CH}_{3}$

$8 \mathrm{R}=\mathrm{H}$<smiles>[R2]c1c(OC)cc([C@H]2c3cc4c(cc3[C@@H](n3cc(CO)nn3)C3COC(=O)[C@H]32)OCO4)cc1OC</smiles>

$11 \mathrm{R}=\mathrm{CH}_{3}$

$12 \mathrm{R}=\mathrm{H}$

SCHEME 1 | The synthesis of podophyllotoxin derivatives (5-12). Reagents and reaction condition: (a) $\mathrm{MeSO}_{3} \mathrm{H}, \mathrm{Nal} \mathrm{CH}_{3} \mathrm{CN} \mathrm{CH}_{2} \mathrm{Cl}_{2}$; then, $\mathrm{H}_{2} \mathrm{O}-\mathrm{Acetone}_{\mathrm{B}} \mathrm{BaCO}$, rt. 90-92\%; (b) $\mathrm{NaN}_{3}-\mathrm{TFA}, \mathrm{CHCl}_{3}, 67-70 \%$; (c) $\mathrm{PPh}_{3}$, THF, then $\mathrm{H}_{2} \mathrm{O}, 72-75 \%$; (d) copper (II) acetate, propargyl alcohol, sodium ascorbate, $t$ - $\mathrm{BuOH}-\mathrm{H}_{2} \mathrm{O}$, THF, rt. $85-89 \%$.

\section{Cell Viability Assay}

Cell viability was evaluated by 3-(4,5-dimethyl-thiazol-2-yl)-2,5diphenyltetrazolium bromide (MTT) assay. Briefly, in each well of a 96-well cell culture plate adherent cells $(100 \mu \mathrm{L})$ with an initial density of $1 \times 10^{5}$ cells $/ \mathrm{mL}$ were seeded and allowed to adhere for $12 \mathrm{~h}$ before a test drug was added. In contrast, suspended cells with the same initial density were seeded just before drug addition. Each tumor cell line was exposed to the test compound at various concentrations in triplicate for $48 \mathrm{~h}$. After the incubation, MTT $(100 \mu \mathrm{g})$ was added to each well, and the incubation continued at $37^{\circ} \mathrm{C}$ for $4 \mathrm{~h}$. The cells were lysed with SDS $(200 \mu \mathrm{L})$ after the removal of the medium. The absorbance of the lysate was measured at $595 \mathrm{~nm}$ by spectrophotometry (microtiter plate reader, Bio-Rad 680). Dose response curves of cell viability were plotted and the $\mathrm{IC}_{50}$ values of test compounds at which $50 \%$ reduction in cell growth were determined.

\section{Cell Apoptosis Assay}

The Annexin V/propidium iodide (PI) detection kit (BD Biosciences, PA, USA) was employed to quantify apoptosis using flow cytometry. H1299 and H1975 cells were seeded in each well of a 12 -well plate at $2.5 \times 10^{5}$ cells/well. After incubation for $24 \mathrm{~h}$, the cells were treated with compound $\mathbf{1 5}$ at $0.5,1$ and $2 \mu \mathrm{M}$ or PPT $(1 \mu \mathrm{M})$ for $24 \mathrm{~h}$. Then, the cells were collected and binding buffer $(100 \mu \mathrm{L})$, FITC annexinV $(5 \mu \mathrm{L})$, and propidium iodide (PI, $10 \mu \mathrm{L}$ ) (eBioscience, San Diego, CA, USA) were added to the cell suspension. The cells were gently vortexed and incubated at room temperature in the dark for $15 \mathrm{~min}$ before measurement by flow cytometry (BD FACSCalibur ${ }^{\mathrm{TM}}$ ) within $1 \mathrm{~h}$.

\section{Western Blotting Analysis}

H1299 and H1975 cell lines were treated with compound 15 at different concentrations in 6-well plates, and then the cells were collected and lysed with lysis buffer. After sonication cells were centrifuged at $14,000 \mathrm{rpm}$ at $4^{\circ} \mathrm{C}$ for $10 \mathrm{~min}$, and total protein was extracted and detected using a bicinchoninc acid (BCA) assay kit. The samples were then separated by $10 \%$ SDS-polyacrylamide gel electrophoresis (PAGE) and then the protein was transferred to nitrocellulose (NC) membranes. The membranes were probed for the following proteins with primary antibodies at $4^{\circ} \mathrm{C}$ overnight: caspase- 3 , cleaved caspase-3, PARP, cleaved PARP, GRP78, CHOP, XBP-1, XBP-1s, and Actin. After washing the membranes with PBST $(\times 1)$, the HRP-conjugated secondary antibodies were added and incubated for $1 \mathrm{~h}$ at room temperature. The membranes were then washed and the HRP was detected using Luminata ${ }^{\mathrm{TM}}$ Forte Western HRP Substrate reagent. The bands of interest were visualized and imaged under chemiluminescent detection using a FluorChem E System (ProteinSimple, San Jose, CA, USA). 

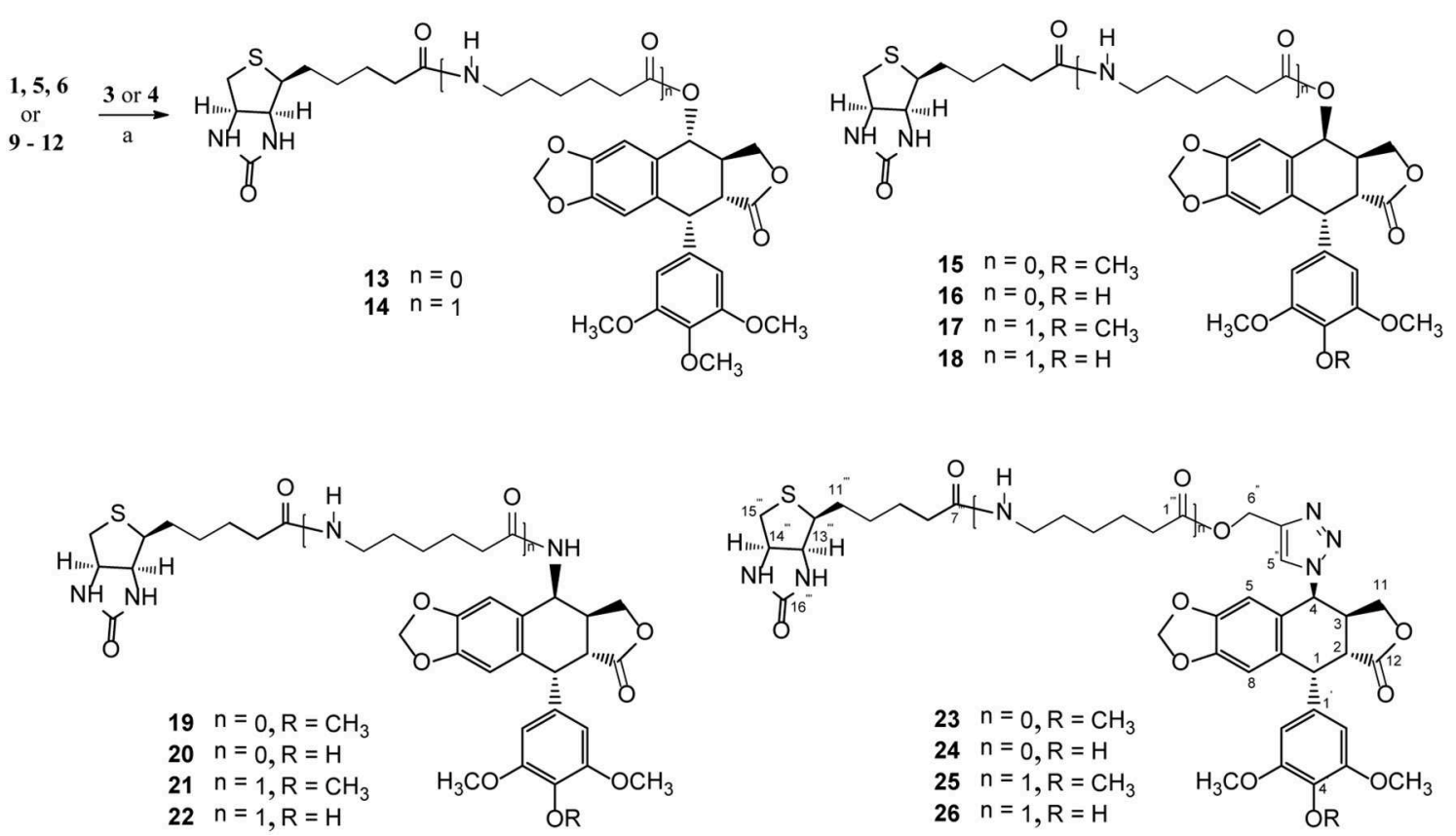

SCHEME 2 | The synthesis of biotinylated podophyllotoxin derivatives (13-26). Reagents and reaction condition: (a) DIC, DMAP, DMF, N2, rt. 39-65\%.

\section{Gene Expression Assay}

H1299 cells were cultured in 12 -well plated at $2.5 \times 10^{5}$ cell/well in the presence of compound $15(0.5,1$, and $2 \mu \mathrm{M})$ for $24 \mathrm{~h}$. Total RNAs present in the cultured cells were extracted using the TransZol ${ }^{\mathrm{TM}}$ Up Reagent (TransGen Biotech, Beijing, China). Gene expression was detected via quantitative real-time PCR (qRT-PCR) and SYBR ${ }^{\circledR}$ Premix EX Taq ${ }^{\text {TM }}$ II (TaKaRa Bio, Otsu, Japan) was used to perform the analysis.

\section{Animal Studies}

All animal studies were conducted in accordance to procedures approved by the Animal Care and Use Committee at China Pharmaceutical University (Jiangsu, China). Forty icr male mice (10-20 g) were provided from the Comparative Medicine Centre of Yangzhou University (Jiangsu, China) and were housed in an SPE animal facility. S180 cancer cells were injected subcutaneously into the right axillaries of icr male mice $\left(4.5-5.0 \times 10^{6}\right.$ cells/spot $)$. The mice were divided randomly into five groups: model; positive control; lowdose; medium-dose; high-dose. All mice of the therapeutic groups were injected intraperitoneally (i.p.) every day, and all mice in the positive control group were injected intravenously (i.v.) on the first day and the fourth day after inoculation. Tumor size was measured with caliper and the volume calculated using the previously reported method (Qin et al., 2015). The weight of the mice and the volume of the tumors were measured every day. At the end of the experiment, the mice were killed and the tumors were isolated and weighed.
TABLE 1 | The in vitro cytotoxic activity $\left(\mathrm{IC}_{50}, \mu \mathrm{M}\right)$ of biotinylated podophyllotoxin derivatives $\mathbf{1 3}-\mathbf{2 6}$ and PPT.

\begin{tabular}{llllll}
\hline \multirow{2}{*}{ compds. } & \multicolumn{5}{c}{ IC $_{\mathbf{5 0}}(\boldsymbol{\mu} \mathbf{M})$} \\
\cline { 2 - 6 } & HL-60 & SMMC-7721 & A-549 & MCF-7 & SW480 \\
\hline $\mathbf{1 3}$ & 0.22 & 0.75 & 0.73 & 1.48 & 1.46 \\
$\mathbf{1 4}$ & 0.18 & 0.65 & 0.58 & 1.26 & 0.96 \\
$\mathbf{1 5}$ & 0.13 & 0.23 & 0.51 & 0.84 & 0.56 \\
$\mathbf{1 6}$ & 0.18 & 0.72 & 1.19 & 8.00 & 0.79 \\
$\mathbf{1 7}$ & 0.19 & 0.71 & 0.80 & 2.97 & $>40$ \\
$\mathbf{1 8}$ & 0.21 & 0.70 & 0.67 & 0.85 & 3.09 \\
$\mathbf{1 9}$ & 13.94 & 19.28 & 28.99 & 17.57 & 27.13 \\
$\mathbf{2 0}$ & 0.46 & 1.40 & 1.34 & 1.60 & 1.25 \\
$\mathbf{2 1}$ & 13.83 & 31.23 & 24.37 & 16.94 & $>40$ \\
$\mathbf{2 2}$ & $>40$ & $>40$ & $>40$ & $>40$ & $>40$ \\
$\mathbf{2 3}$ & 10.38 & 19.21 & 17.33 & 23.46 & 39.9 \\
$\mathbf{2 4}$ & 3.64 & $>40$ & 30.67 & $>40$ & $>40$ \\
$\mathbf{2 5}$ & $>40$ & $>40$ & $>40$ & $>40$ & $>40$ \\
$\mathbf{2 6}$ & $>40$ & $>40$ & $>40$ & $>40$ & $>40$ \\
$\mathbf{P P T}$ & $<0.064$ & 4.13 & $<0.064$ & $<0.064$ & 9.42 \\
Etoposide & 0.31 & 8.12 & 11.92 & 32.82 & 17.11 \\
Cisplatin & 1.17 & 6.43 & 9.24 & 15.86 & 13.42 \\
\hline
\end{tabular}

\section{Molecular Docking Studies}

The crystal structure of Top-II (code ID: 3QX3) (Wu et al., 2011) was obtained in Protein Data Bank after eliminating the inhibitor and water molecules. The missing atoms were added by Sybyl-X 2.0 molecular modeling. The kinds of atomic charges 
TABLE 2 | IC $\mathrm{IC}_{50}$ values of compound $\mathbf{1 5}$ in twelve cancer cell lines.

\begin{tabular}{lll}
\hline Cell line & Type & $\mathbf{I C}_{\mathbf{5 0}}(\boldsymbol{\mu} \mathbf{M})$ \\
\hline H460 & Lung cancer & 6.53 \\
H1975 & Lung cancer & 3.31 \\
H1299 & Lung cancer & 0.86 \\
LS174T & Colon cancer & 3.30 \\
HCT-116 & Colon cancer & 1.08 \\
HT-29 & Colon cancer & 1.39 \\
BGC-823 & Stomach cancer & 4.41 \\
MGC-803 & Stomach cancer & 4.45 \\
SKBR3 & Breast cancer & 5.93 \\
T47D & Breast cancer & $>10$ \\
Bel-7402 & Hepatoma & 10.0 \\
Hela & Cervical cancer & 1.04 \\
\hline
\end{tabular}

were taken as Kollman-united-atom (Weiner et al., 1984) for the macromolecule and Gasteiger-Marsili (Gasteiger and Marsili, 1980) for the inhibitor. To find the binding mode of compound 15 to the active site of Top-II, the advanced docking program Autodock Tools v1.56 (Morris et al., 1998) was used for grid and docking. The enzyme structure was used as an input for the AutoGrid program. AutoGrid performed pre-calculated atomic affinity grid maps for each atom type in the ligand plus a separate desolvation map, and a separate desolvation map present in the substrate molecule. Docking parameters were set as the default values except docking runs was set to 100 on AutoGrid v4.01 and AutoDock v4.01.

\section{Statistical Analysis}

All data are presented as the means $\pm \mathrm{SD}(n=3)$. Significance was calculated using Student's $t$-test or one-way ANOVA. $P<$ 0.05 was considered statistically significant. All statistical analyses were performed with the GraphPad Prism 5.0 (San Diego, CA, USA).

\section{RESULTS AND DISCUSSION}

\section{Chemical synthesis}

Podophyllotoxin (PPT) served as the starting material for the preparation of all the derivatives. The incorporation of the azido, amino, and triazolyl groups at the 4-position of PPT followed standard procedures (Scheme 1). PPT was regioselectively demethylated with methanesulfonic acid and sodium iodide in dichloromethane $\left(\mathrm{CH}_{2} \mathrm{Cl}_{2}\right)$ followed by weak basic hydrolysis (water-acetone, barium carbonate) to give $4^{\prime}$ $O$-demethylepipodophyllotoxin $\mathbf{6}$ by means of a previously described procedure (Kamal et al., 2000). When the reaction was carried out in acetonitrile $\left(\mathrm{CH}_{3} \mathrm{CN}\right)$ as a solvent, $4 \beta$ epipodophyllotoxin $\mathbf{5}$ was synthesized as product. Compound 5 and $\mathbf{6}$ were converted into the corresponding $4 \beta$-azides 7 and 8 by reaction with sodium azide and trifluoroacetic acid $\left(\mathrm{NaN}_{3}\right.$-TFA) in chloroform $\left(\mathrm{CHCl}_{3}\right)$ according to the known procedure (Hansen et al., 1993). The $4 \beta$-azides 7 and 8 were converted to $4 \beta$-amino substituted 9 and 10 by treatment with triphenylphosphine $\left(\mathrm{Ph}_{3} \mathrm{P}\right)$ and water overnight at $25^{\circ} \mathrm{C}$ as previously reported (Coleman and Kong, 1998). In addition, the $4 \beta$-triazole compounds of $\mathbf{1 1}$ and 12 were prepared in $85-89 \%$ yield by the reaction of 7 and 8 , respectively, with 2-propyn-1-ol using copper (II) acetate and sodium ascorbate as promoters in tert-butanol and water $(t$ $\mathrm{BuOH}-\mathrm{H}_{2} \mathrm{O}, 1: 1$ ) at room temperature (Tae et al., 2010). Finally, biotin (3)/6-biotinylaminocaproic acid (4) and those podophyllotoxin derivatives $(\mathbf{1}, \mathbf{5}, \mathbf{6}$, and $\mathbf{9 - 1 2})$ were coupled via an ester or amide bond. As shown in Scheme 2, biotin (3)/6biotinylaminocaproic acid (4) reacted with compounds 1, 5, 6, and 9-12 in the presence of diisopropylcarbodiimide (DIC) and 4 - $N, N$-dimethylaminopyridine (DMAP) at room temperature to afford the target compounds 13-26 in 39-65\% yields.

All the products were structurally confirmed by ${ }^{1} \mathrm{H}$ and ${ }^{13} \mathrm{C}$-NMR spectroscopies, as well as low resolution and high resolution mass spectrometry in electrospray ionization mode (ESI-MS and HRESI-MS). The proton and carbon-13 NMR data of these compounds were compared with those of podophyllotoxin. The configuration of C-4 in compounds 13-26 was assigned based on the coupling constant between $\mathrm{H}-3$ and $\mathrm{H}-$ $4\left(J_{3,4}\right)$. Typically, compounds with C- $4 \beta$-substitution have $J_{3,4}<$ $5.0 \mathrm{~Hz}$ as a result of $\mathrm{H}-3$ and $\mathrm{H}-4$ in cis relationship. The protons at C-4 of compounds 19-21 appear as a singlet. On the other hand, compounds with C- $4 \alpha$-substitution have $J_{3,4}>6.0 \mathrm{~Hz}$ because H-4 is trans to H-3 (Fred Brewer et al., 1979; Belen'kiib and Schinazi, 1994). In the ${ }^{13} \mathrm{C}-\mathrm{NMR}$ spectra, the C-4 of these derivatives produces a characteristic signal between 61.1 and 71.3 ppm. The triazole ting in 23-26 was readily confirmed by its $\mathrm{C}^{5 \text { " }}$-H signal ( $\left.\delta 7.36-7.74 \mathrm{ppm}\right)$ in the aromatic region in the ${ }^{1} \mathrm{H}$ NMR spectra, which was further supported by the characteristic carbon signals at around $123 \mathrm{ppm}$ in the ${ }^{13} \mathrm{C}-\mathrm{NMR}$ spectra.

\section{Biology}

\section{Cytotoxicity and Structure-Activity Relationship}

The cytotoxicity of all biotinylated podophyllotoxin derivatives 13-26 was tested with the following cancer cell lines: SW480 (colon cancer), MCF-7 (breast cancer), A-549 (lung cancer), SMMC-7721 (hepatoma), and HL-60 (leukemia), Podophyllotoxin (PPT), etoposide, and cisplatin were included for study as control drugs. The $\mathrm{IC}_{50}$ values obtained from MTT assay are presented in Table 1. Most compounds possessed high level of cytotoxicity against all five cancer cell lines (Table 1) and were more active than etoposide which is an antitumor agent currently in clinical use.

Biotinylated podophyllotoxin derivatives are prepared by linking a biotinylating agent, biotin (3) or 6biotinylaminocaproic acid (4), via an ester bond, an amide bond, or a trizolyl moiety. Those compounds with an ester linkage (13-18) display potent cytotoxicity with $\mathrm{IC}_{50}$ values in sub- $\mu \mathrm{M}$ to low $\mu \mathrm{M}$ (except compound $\mathbf{1 7}$ against SW480 cell line). Compounds $\mathbf{1 3}$ and $\mathbf{1 4}$ are esters of podophyllotoxin while 15-18 are esters of 4-epipodophyllotoxin, and their similar potency of activity indicates that the cytotoxic activity of these compounds is not much affected by the configuration of C-4. Among the synthesized compounds, compound $\mathbf{1 5}$ is the most active one with $\mathrm{IC}_{50}$ ranged from 0.13 to $0.84 \mu \mathrm{M}$. Compound 15 also exhibits higher activity than PPT in both 

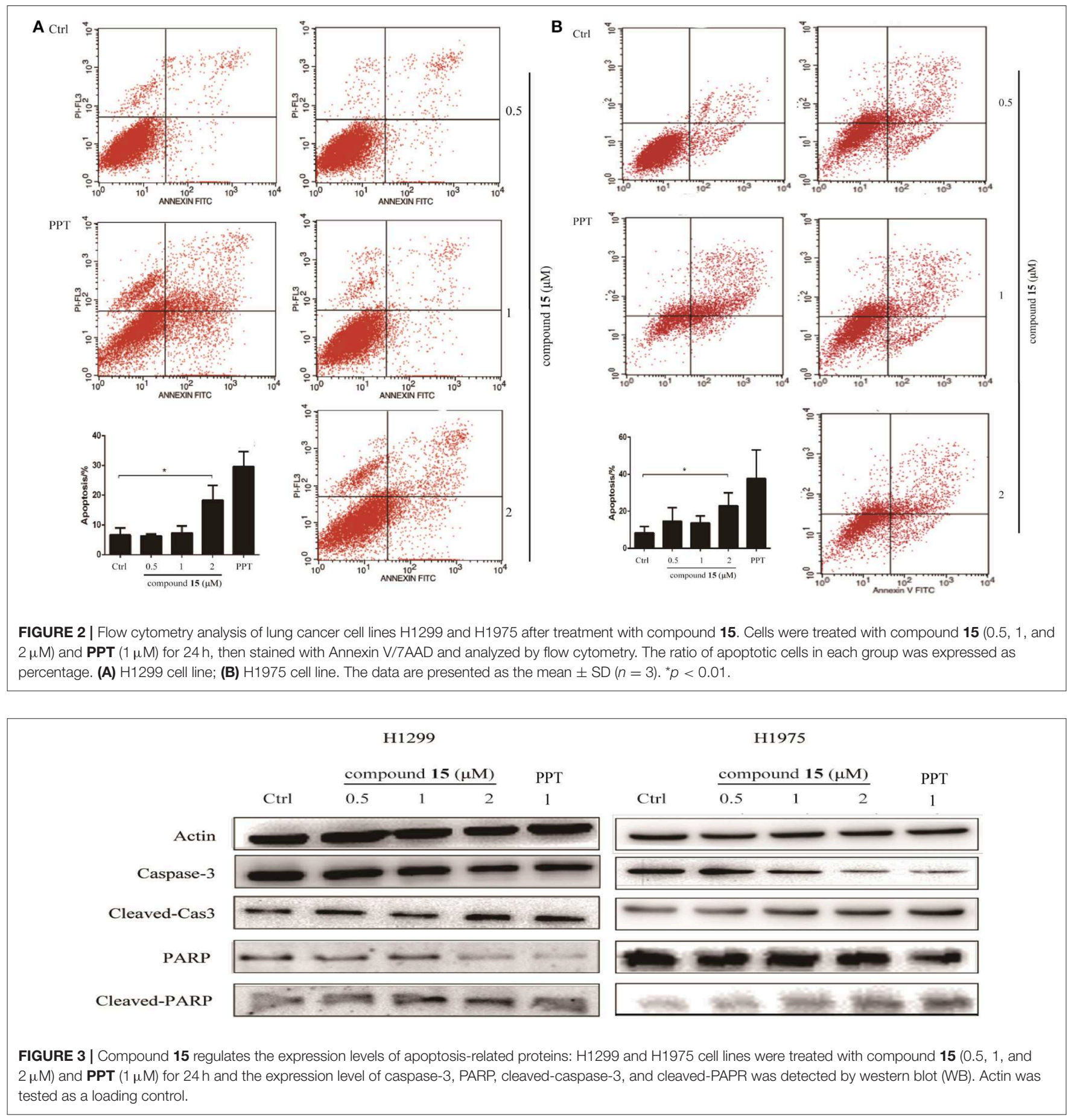

SMMC-7721 and SW480 cell lines, with PPT having $\mathrm{IC}_{50}$ of 4.13 and $9.42 \mu \mathrm{M}$, respectively.

Compounds with an amide linkage (19-22) or a triazolyl moiety (23-26) show weaker cytotoxicity to all tested cell lines. Most of these compounds display moderate $\left(\mathrm{IC}_{50}>10.36 \mu \mathrm{M}\right)$ to very weak activity $\left(\mathrm{IC}_{50}>40 \mu \mathrm{M}\right.$; except compound 20 , as well as compound 24 against HL-60 cell line). The 6-aminocaproic acid linking spacer present in C-4-substituent can affect the cytotoxic potency of these compounds but not in a uniform way. For example, compounds lacking the linking spacer (15, 20, and 23) show higher activity than their counterparts bearing the linking spacer $(\mathbf{1 7}, \mathbf{2 2}$, and 25) in all cell lines tested. In contrast, compound 13 (lacking the linking spacer) is less active than 14 (bearing the linking spacer). In most cases, the effect of 6-aminocaproic acid linking spacer on the cytotoxic potency of these compounds are relatively small except for the pair of compounds $\mathbf{1 5}$ and $\mathbf{1 7}$ in SW480 cell line ( $\mathrm{IC}_{50} 0.56$ and $>40 \mu \mathrm{M}$, respectively). However, it is very interesting to 


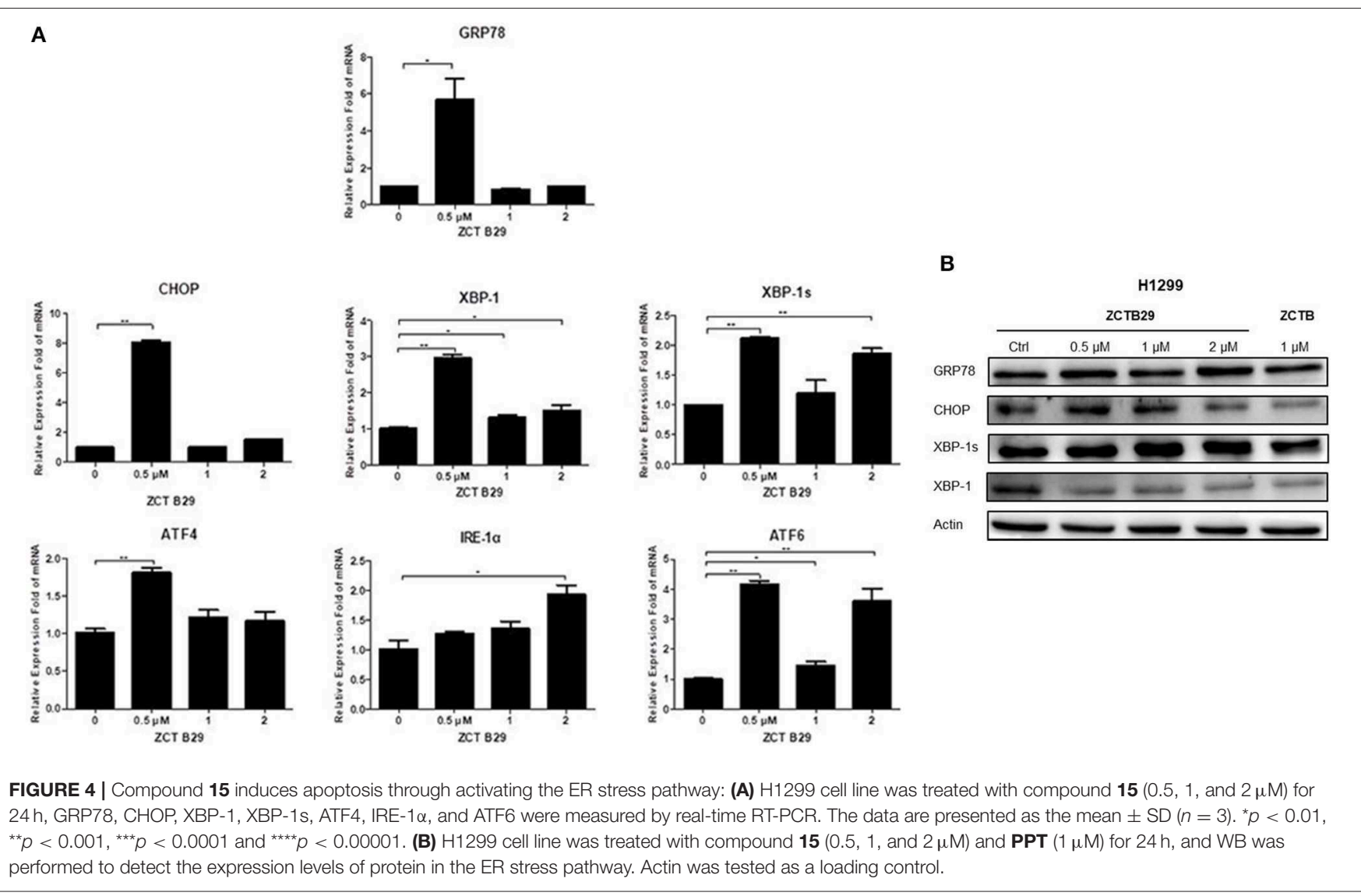

note that compound 20 (lacking the linking spacer, $\mathrm{IC}_{50} 0.46-$ $1.60 \mu \mathrm{M}$ ) is significantly more potent than 22 (bearing the linking spacer, $\mathrm{IC}_{50}>40 \mu \mathrm{M}$ ) in all cell lines tested. Furthermore, compound 19, the 4'-O-methylated form of 20 , shows much lower activity $\left(\mathrm{IC}_{50} 13.94-28.99 \mu \mathrm{M}\right)$ than 20, which is in good agreement with our earlier observation that the 4'-O-methylation can significantly affect the anticancer activity of podophyllotoxin derivatives (Zi et al., 2015, 2017).

\section{Compound 15 Inhibits the Growth of Cancer Cell Lines}

To further identify the anticancer effect and tumor selectivity of compound 15, we treated 12 more human cancer cell lines with compound 15, which included lung cancer (H460, H1975, H1299), colon cancer (LS174T, HCT-116, HT-29), stomach cancer (BGC-823, MGC-803), breast cancer (SKBR3, T47D), hepatoma (Bel-7402), and cervical cancer (Hela). MTT assay was employed to provide the $\mathrm{IC}_{50}$ values of compound $\mathbf{1 5}$ against all these tumor cell lines as shown in Table 2. H1299 cell line was most sensitive toward compound $15\left(\mathrm{IC}_{50}=0.86 \mu \mathrm{M}\right)$. For most other cancer cell lines, compound $\mathbf{1 5}$ showed potent anticancer activity with $\mathrm{IC}_{50}$ values in $\mu \mathrm{M}$ range. In order to test whether compound 15 can favorably target cancer cells over normal cells, the growth inhibitory effect of 15, in comparison with PPT, on a normal human bronchial epithelial cell line (BEAS-2B) was evaluated. The $\mathrm{IC}_{50}$ value was found to be $3.75 \mu \mathrm{M}$ for 15 and $0.85 \mu \mathrm{M}$ for PPT against BEAS-2B cells (see Table S1).
Comparing with its $\mathrm{IC}_{50}$ values in Table $1(0.13-0.84 \mu \mathrm{M})$ and Table 2 against various cancer cell lines, compound $\mathbf{1 5}$ does show some selectivity against certain tested cancer cell lines over the normal cells (BEAS-2B).

\section{Compound 15 Induces Apoptosis in the H1299 and H1975 Cell Lines}

Given that compound $\mathbf{1 5}$ exhibits broad spectrum inhibitory activity of cancer cell growth, we studied further the capacity of compound $\mathbf{1 5}$ in the induction of cell death through apoptosis. Lung cancer cells (H1299 and H1975) were treated with compound $\mathbf{1 5}$ and analyzed by flow cytometry after being stained with Annexin V/7AAD. Compound $\mathbf{1 5}$ at concentration of $2 \mu \mathrm{M}$ increased significantly both H1299 and H1975 cells undergoing apoptosis when compared with the untreated control (Figure 2).

\section{Compound 15 Regulates the Expression Levels of Apoptosis-Related Protein}

It has been recognized that caspase- 3 and PARP (poy ADP ribose polymerase) is a critical initiator and executioner of apoptosis (Hensley et al., 2013). H1299 and H1975 cells were treated with compound 15 at the concentration of $0.5,1,2$ or $\mu \mathrm{M}$ for $24 \mathrm{~h}$ and the expression level of caspase-3, PARP, cleaved-caspase3 , and cleaved-PARP was monitored using western blot. The treatment of both H1299 and H1975 cell lines with compound 15 resulted in an increased expression level of cleaved-caspase-3 and 
A<smiles></smiles>

C

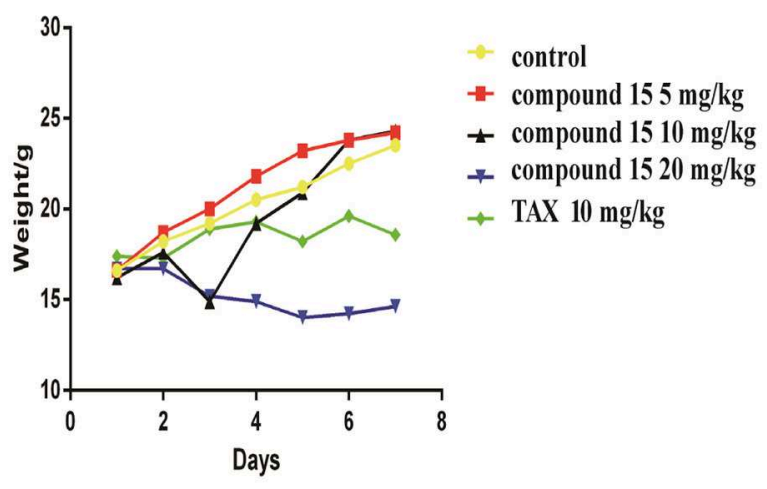

B

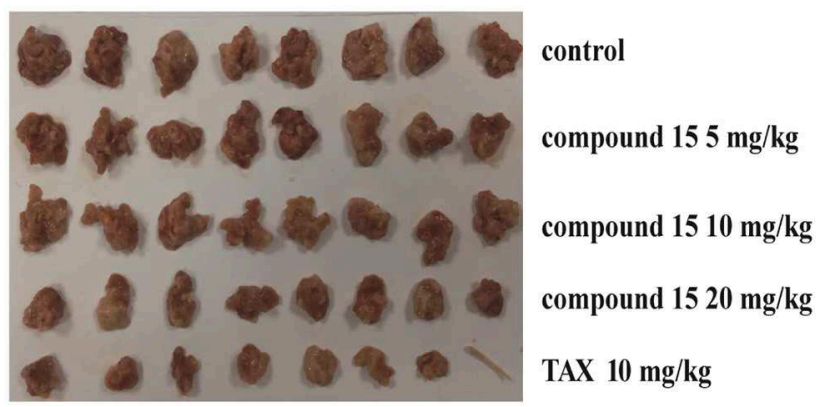

D

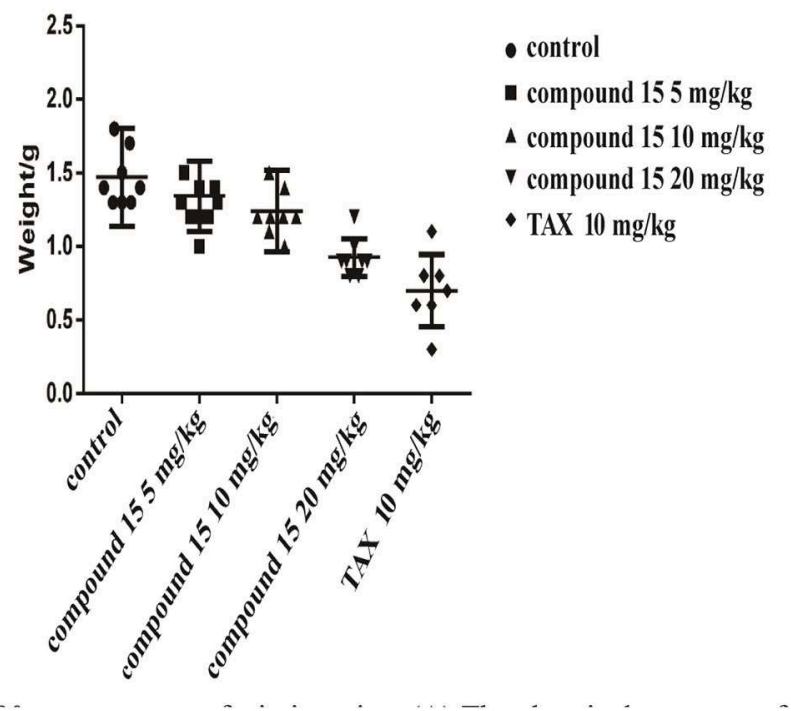

FIGURE 5 | Compound $\mathbf{1 5}$ significantly inhibits the growth of $\mathbf{S 1 8 0}$ tumor xenografts in icr mice: (A) The chemical structure of compound 15. (B) Tumors collected at the end of the treatment (day 7). (C) Mouse's body weights were weighed for those treated with compound 15 (5, 15, $20 \mathrm{mg} / \mathrm{kg})$ compared to TAX control (10 mg/kg) TAX: Taxol. (D) Tumor weights were presented for the groups treated with compound 15 (5, 15, $20 \mathrm{mg} / \mathrm{kg})$.

cleaved-PARP in a dose-dependent manner (Figure 3). At the same time, the expression level of caspase- 3 and PARP decreased, indicating that the treatment led to the activation of caspase- 3 and the deactivation of PARP and ultimately apoptosis. These data confirmed that the compound $\mathbf{1 5}$ exhibits its anticancer activity through induction of apoptosis in both H1299 and H1975 cell lines.

\section{Compound 15 Induces Apoptosis Through Activating IRE1 $\alpha$, a Key Mediator in the Endoplasmic Reticulum (ER) Stress Pathway}

Many studies have indicated that endoplasmic reticulum (ER) stress activates the unfolded protein response (UPR), through which tumor cells can become resistant to chemotherapeutic agents (Cheng et al., 2014). PKR-like ER kinase (PERK), inostitolrequiring transmembrane kinase and endonuclease $1 \alpha$ (IRE- $1 \alpha)$, and activating transcription factor 6 (ATF6) are three primary UPR sensors that lead to distinct downstream signaling pathways (Ron and Walter, 2007). Therefore, we next studied the possible involvement of compound $\mathbf{1 5}$ in the activation of the ER stress pathway. H1299 cell line was treated with compound $\mathbf{1 5}$ at the concentration of $0.5,1$, or $2 \mu \mathrm{M}$ for $24 \mathrm{~h}$ and the mRNA expression level of stress related proteins (GRP78, CHOP, XBP1, XBP-1s, ATF4, IRE-1 $\alpha$, and ATF6) in ER was analyzed (Figure 4A). Interestingly, the mRNA level of all these proteins except IRE- $1 \alpha$ was dramatically increased upon the treatment of compound 15 at $0.5 \mu \mathrm{M}$. The effect of $\mathbf{1 5}$ at other concentrations ( 1 or $2 \mu \mathrm{M}$ ) on the mRNA expression level of these proteins was less significant or negligible. In the case of IRE- $1 \alpha$, the expression level of mRNA increased by the treatment of $\mathbf{1 5}$ in a dose-dependent manner, suggesting that IRE-1 $\alpha$ might play a crucial role in compound 15 -induced apoptosis. We further examined the expression level of a number of these proteins (GRP78, CHOP, XBP-1, and XBP-1s) related to ER 

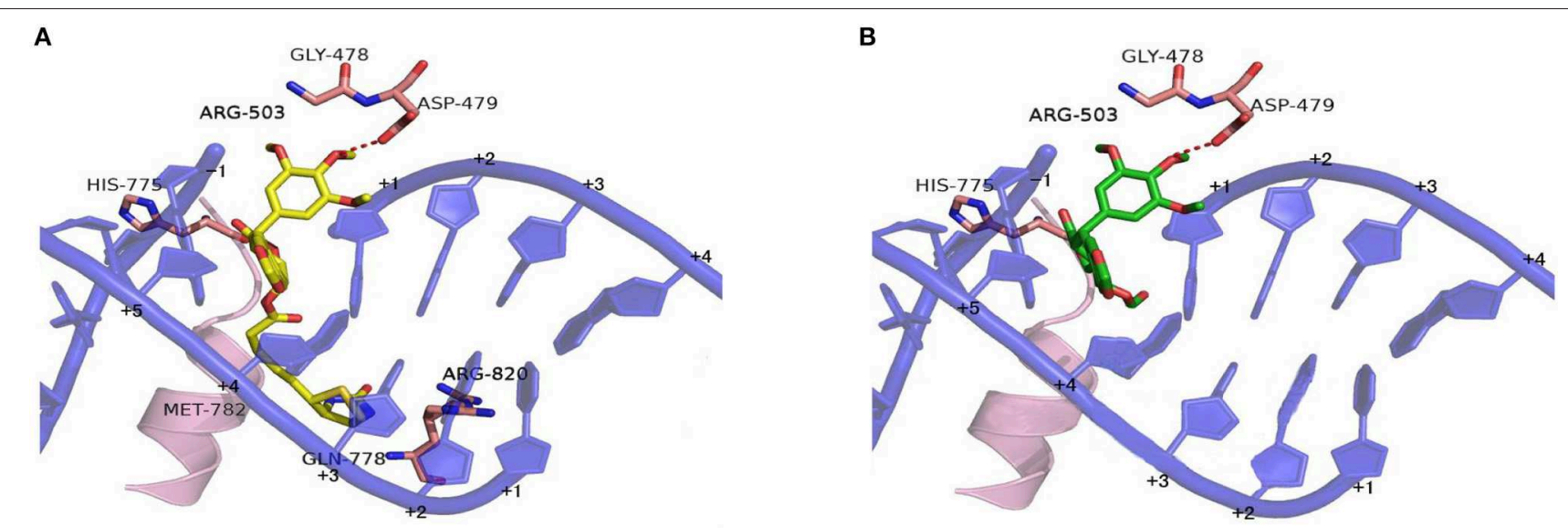

FIGURE 6 | Proposed binding models of compounds $\mathbf{1 5}$ and PPT to the ATPase domain of Topoisomerase-II. Based on the X-ray co-crystal structure of Top-II in complex with Etoposide. (A) Binding mode of compound 15 with Topoisomerase-II. (B) Binding mode of PPT with Topoisomerase-II.

stress (Figure 4B). Compound 15 significantly up-regulated the expression of GRP78 and XBP-1s, and down-regulated the expression of $\mathrm{CHOP}$ and $\mathrm{XBP}-1$.

\section{Compound 15 Significantly Inhibits the Growth of S180 Tumor Xenografts in Icr Mice}

Since compound $\mathbf{1 5}$ suppressed lung cancer cell proliferation in vitro, we further investigated its ability to suppress the growth of S180 tumor xenografts in icr mice (Figure 5). As shown in Figure 5, compound $\mathbf{1 5}(5,15$, or $20 \mathrm{mg} / \mathrm{kg})$ suppressed the growth of $\mathrm{S} 180$ xenografts over the course of 7 days (Figure 5A) comparing to the Taxol ${ }^{\circledR}$ control $(10 \mathrm{mg} / \mathrm{kg})$ and the inhibition rates of compound 15 were 8.8, 15.7, and $37.7 \%$ (Figure 5D), respectively. Tumors were collected at the end of the experiment (Figure 5B) and the tumor weights were measured. The data showed that compound 15 significantly decreased tumor weight when compared to the untreated control, indicating that 15 effectively inhibited the growth S180 tumor xenograft. In addition, compound $\mathbf{1 5}$ did not cause mice to die and did not affect mouse body weight significantly at a dose of up to $20 \mathrm{mg} / \mathrm{kg}$ (Figure 5C).

\section{Docking Studies}

Based on the X-ray crystal structure of Topoisomerase-II inhibitors bound to the ATPase domain of Topo-II (PDB: 3QX3) (Wu et al., 2011), the binding mode between TopoisomeraseII and 15 or PPT was established by autodocking (Figure 6). Compound 15 binds Topo-II between the base pairs immediately flanking the two cleaved scissile phosphates (Figure 6A). Its polycyclic podophyllotoxin core (rings A to D) sits between base pairs, while the biotin side chain and the E ring protrude toward the DNA major and minor grooves, respectively. All parts of the podophyllotoxin core contribute to drug-DNA interaction by being located between base pairs. The $\mathrm{E}$ ring is anchored by both interacting with GLY-478, ASP-479, and ARG503 residues of the enzyme and being sandwiched between R503 and the deoxyribose ring of the +1 nucleotide. Compared to PPT (Figure 6B), compound 15 shows additional hydrophobic interaction with GLN-778 and ARG-820 residues through the biotin moiety. The biotin moiety in $\mathbf{1 5}$ provides an additional hydrophobic moiety and multiple H-bond donors/acceptor, which allows the molecule to interact more favorably with TopoII and might lead to improved selectivity.

\section{Chemical Stability Investigation}

The chemical stability of compound $\mathbf{1 5}$ in aqueous phase was investigated together with podophyllotoxin (PPT, 1) for comparison. The results indicate that compound $\mathbf{1 5}$ degrades slowly under the physiological condition $\left(37 \pm 1^{\circ} \mathrm{C}, \mathrm{pH} 7.0\right)$ with $70 \%$ material remaining after $12 \mathrm{~h}$ (see Figures S1-S3). A similar stability profile was observed for PPT with 75\% material remaining after $12 \mathrm{~h}$.

\section{CONCLUSION}

In summary, a series of biotinylated podophyllotoxin derivatives (13-26) were designed, synthesized, and evaluated for cytotoxicity against five tumor cell lines (HL-60, SMMC7721, A-549, MCF-7, and SW480) by using MTT assay. Among them, compound 15 showed the highest anticancer activity with its $\mathrm{IC}_{50}$ values at $0.13-0.84 \mu \mathrm{M}$. Preliminary structure-activity relationship (SAR) analysis indicated that derivatives bearing an amide or triazolyl linking moiety showed weaker activity than those with an ester linkage. The 6-aminocaproic acid linking spacer affected the cytotoxic potency of these compounds in an ununiform manner. Compound $\mathbf{1 5}$ also reduced the expression levels of caspase- 3 and PARP. Importantly, the pro-apoptotic activity of compound $\mathbf{1 5}$ in H1299 cell line was mediated by the transcription of IRE- $1 \alpha$, which plays an important role in the endoplasmic reticulum stress pathway. Finally, compound 15 at a dose of $20 \mathrm{mg} / \mathrm{kg}$ suppressed the growth of S180 tumor xenografts in icr mice significantly. Molecular docking studies suggested that compound $\mathbf{1 5}$ could bind well with the ATPase domain of Topoisomerase-II. Continuing studies to substantiate 
the further development of compound $\mathbf{1 5}$ as an anticancer agent are underway in our laboratory and will be reported in due course.

\section{DATA AVAILABILITY}

This manuscript contains previously unpublished data. The name of the repository and accession number are not available.

\section{AUTHOR CONTRIBUTIONS}

$\mathrm{JZ}, \mathrm{J}-\mathrm{MH}$, and $\mathrm{Z}-\mathrm{HJ}$ designed and guided this study. C-TZ and F-WD conducted the chemical synthesis. LY and YL performed the cell assay. $\mathrm{YH}$ and F-QX participated in the cell assay. S-TY, Y-SG, and S-YF performed animal experiments. YJ performed molecular docking. LS and Z-TD performed the SPR binding assay. LS, Z-TD, and J-MH

\section{REFERENCES}

Belen'kiib, M. S., and Schinazi, R. F. (1994). Multiple drug effect analysis with confidence interval. Antiviral Res. 25, 1-11. doi: 10.1016/0166-3542(94)90089-2

Bermejo, A., Figadere, B., Zafra-Polo, M. C., Barrachina, I., Estornell, E., and Cortes, D. (2005). Acetogenins from Annonaceae: recent progress in isolation, synthesis and mechanisms of action. Nat. Prod. Rep. 22, 269-303. doi: 10.1039/b500186m

Bonifácio, B. V., da Silva, P. B., dos Santos Ramos, M. A., Silveira Negri, K. M., Bauab, T. M., and Chorilli, M. (2014). Nanotechnology-based drug delivery systems and herbal medicines: a review. Int. J. Nanomed. 9, 1-15. doi: 10.2147/IJN.S52634

Bromberg, K. D., Burgin, A. B., and Osheroff, N. (2003). A two-drug model for etoposide action against human topoisomerase Ii $\alpha$. J. Biol. Chem. 278, 7406-7412. doi: 10.1074/jbc.M212056200

Chen, J. Y., Chen, S. Y., Zhao, X. R., Kuznetsova, L. V., Wong, S. S., and Ojima, I. (2008). Functionalized single-walled carbon nanotubes as rationally designed vehicles for tumor-targeted drug delivery. J. Am. Chem. Soc. 130, 16778-16785. doi: 10.1021/ja805570f

Chen, S. Y., Zhao, X. R., Chen, J. Y., Chen, J., Kuznetsova, L., Wong, S. S., et al. (2010). Mechanism-based tumor-targeting drug delivery system. Validation of efficient vitamin receptor-mediated endocytosis and drug release. Bioconjugate Chem. 21, 979-987. doi: 10.1021/bc9005656

Cheng, X., Liu, H., Jiang, C. C., Fang, L., Chen, C., Zhang, X. D., et al. (2014). Connecting endoplasmic reticulum stress to autophagy through IRE1/JNK/beclin-1 in breast cancer cells. Int. J. Mol. Med. 34, 772-781. doi: 10.3892/ijmm.2014.1822

Coleman, R. S., and Kong, J. S. (1998). Stereocontrolled synthesis of the fully elaborated aziridine core of the azinomycins. J. Am. Chem. Soc. 120, 3538-3539. doi: $10.1021 /$ ja9801386

Desbene, S., and Giorgi-Renault, S. (2002). Drugs that inhibit tubulin polymerization: the particular case of podophyllotoxin and analogues. Curr. Med. Chem. Anti-Cancer Agents 2, 71-90. doi: 10.2174/1568011023354353

Dharap, S. S., Wang, Y., Chandna, P., Khandare, J. J., Qiu, B., Gunaseelan, S., et al. (2005). Tumor-specific targeting of an anticancer drug delivery system by LHRH peptide. Proc. Natl. Acad. Sci. U.S.A. 102, 12962-12967. doi: $10.1073 /$ pnas.0504274102

Fred Brewer, C., Loike, J. D., Horwitz, S. B., Sternlicht, H., and Gensler, W. J. (1979). Conformational analysis of podophyllotoxin and its congeners. Structureactivity relationship in microtubule assembly. J. Med. Chem. 22, 215-221. doi: 10.1021/jm00189a001

Fulda, S. (2010). Modulation of apoptosis by natural products for cancer therapy. Planta Med. 76, 1075-1079. doi: 10.1055/s-0030-1249961 contributed reagents, materials, and analysis tools. C-TZ and Y-SG analyzed the data. C-TZ, LY, Y-SG, Z-HJ, and J-MH wrote the manuscript. All authors read and approved the final manuscript.

\section{ACKNOWLEDGMENTS}

This work was financially supported by grants from Yunnan Provincial Science and Technology Department (Nos. 2017ZF003, 2015FB168, 2015HB093, and 2018HA001), and the National Nature Science Foundation of China (No. 21602196).

\section{SUPPLEMENTARY MATERIAL}

The Supplementary Material for this article can be found online at: https://www.frontiersin.org/articles/10.3389/fchem. 2019.00434/full\#supplementary-material

Gasteiger, J., and Marsili, M. (1980). Iterative partial equalization of orbital electronegativity-a rapid access to atomic charges. Tetrahedron 36, 3219-3228. doi: 10.1016/0040-4020(80)80168-2

Guaragna, A., Chiaviello, A., Paolella, C., D’Alonzo, D., and Palumbo, G. (2012). Synthesis and evaluation of folate-based chlorambucil delivery systems for tumor-targeted chemotherapy. Bioconjug Chem. 23, 84-96. doi: 10.1021/bc200410d

Hansen, H. F., Jesen, R. B., Willumsen, A. M., Norsko-Lauritsen, N., Ebbesen, P., Nielen, P. E., et al. (1993). New compounds related to podophyllotoxin and congeners: Synthesis, structure elucidation and biological testing. Acta Chem. Scand. 47, 1190-1200. doi: 10.1002/chin.199416284

Hensley, P., Mishra, M., and Kyprianou, N. (2013). Targeting caspases in cancer therapeutics. Bio. Chem. 394, 831-843. doi: 10.1515/hsz-2013-0128

Hojjat-Farsangia, M., Moshfegha, A., Daneshmanesha, A. H., Khana, A. S., and Mikaelssona, E., Österborga, A. et al. (2014). The receptor tyrosine kinase ROR1-An oncofetal antigen for targeted cancer therapy. Semin. Cancer Biol. 29, 21-31. doi: 10.1016/j.semcancer.2014.07.005

Holschneider, C. H., Johnson, M. T., Knox, R. M., Rezai, A., Ryan, W. J., and Montz, F. J. (1994). Bullatacin-in vivo and in vitro experience in an ovarian Cancer model. Cancer Chemother. Pharmacol. 34, 166-170. doi: 10.1007/BF00685935

Jardine, I. (1980). "Podophyllotoxins," in Anticancer Agents Based on Natural Product Models, eds J. M. Cassady and J. D. Douros (New York, NY: Academic Press), 319-351.

Kamal, A., Laxman, N., and Ramesh, G. (2000). Facile and efficient one-pot synthesis of $4 \beta$-arylaminopodophyllotoxins: synthesis of DNA topoisomerase II inhibitors (NPF and W-68). Bioorg. Med. Chem. Lett. 10, 2059-2062. doi: 10.1016/S0960-894X(00)00407-8

Lambert, J. M., and Berkenblit, A. (2018). Antibody-drug conjugates for cancer treatment. Annu. Rev. Med. 69, 191-207. doi: 10.1146/annurev-med-061516-121357

Leamon, C. P. (2008). Folate-targeted drug strategies for the treatment of cancer. Curr. Opin. Investig. Drugs 9, 1277-1286. doi: 10.2174/0929867 08786848505

Liu, Y. Q., Yang, L., and Tian, X. (2007). Podophyllotoxin: Current Perspectives. Curr. Bioact. Compd. 3, 37-66. doi: 10.2174/157340707780 126499

Lu, Y. J., and Low, P. S. (2012). Folate-mediated delivery of macromolecular anticancer therapeutic agents. Adv. Drug Deliv. Rev. 64, 342-352. doi: 10.1016/j.addr.2012.09.020

Mastrobattista, E., Koning, G. A., and Storm, G. (1999). Immunoliposomes for the targeted delivery of antitumor drugs. Adv. Drug Deliv. Rev. 40, 103-127. doi: 10.1016/S0169-409X(99)00043-5 
Morris, G. M., Goodsell, D. S., Halliday, R. S., Huey, R., Hart, W. E., Belew, R. K., et al. (1998). Automated docking using a Lamarckian genetic algorithm and an empirical binding free energy function. J. Comput. Chem. 19, 1639-1662.

Ojima, I. (2008). Guided molecular missiles for tumor-targeting chemotherapycase studies using the second-generation taxoids as warheads. Acc. Chem. Res. 41, 108-119. doi: 10.1021/ar700093f

Ojima, I., Zuniga, E. S., Berger, W. T., and Seitz, J. D. (2012). Tumor-targeting drug delivery of new-generation toxoids. Future Med. Chem. 4, 33-50. doi: $10.4155 /$ fmc. 11.167

Peer, D., Karp, J. M., Hong, S., Farokhzad, O. C., Margalit, R., and Langer, R. (2007). Nanocarriers as an emerging platform for cancer therapy. Nat. Nanotechnol. 2, 751-760. doi: 10.1038/nnano.2007.387

Qiao, W. J., Cheng, H. Y., Li, C. Q., Jin, H., Yang, S. S., Li, X., et al. (2011). Identification of pathways involved in paclitaxel activity in cervical cancer. Asian Pac. J. Cancer Prev. 12, 99-102. doi: 10.1097/01.cad.0000390767.85658.83

Qin, J. Y., Zhou, Z. M., Chen, W. L., Wang, W. L., Zhang, H. L., Ge, G. Z., et al. (2015). BAP1 promotes breast cancer cell proliferation and metastasis by deubiquitinating KLF5. Nat. Commun. 6:8471. doi: 10.1038/ncomms9471

Reddy, P. B., Paul,1, D. V., Agrawal, S. K., Saxena, A. K., and Kumar, H. M. S., Qazi, G.N. (2008). Design, synthesis, and biological testing of $4 \beta$-[(4-Substituted)1,2,3-triazol-1-yl]podophyllotoxin Analogues as Antitumor Agents. Archiv. Pharm. 341, 126-131. doi: 10.1002/ardp.200700116

Ron, D., and Walter, P. (2007). Signal integration in the endoplasmic reticulum unfolded protein response. Nat. Rev. Mol. Cell Bio. 8, 519-529. doi: $10.1038 / \mathrm{nrm} 2199$

Russell-Jonesa, G., McTavisha, K., McEwana, J., Riceb, J., and Nowotnikb, D. (2004). Vitamin-mediated targeting as a potential mechanism to increase drug uptake by tumours. J. Inorg. Biochem. 98, 1625-1633. doi: 10.1016/j.jinorgbio.2004.07.009

Sawant, R. R., Sawant, R. M., and Torchilin, V. P. (2008). Mixed PEG-PE/vitamin E tumor-targeted immunomicelles as carriers for poorly soluble anti-cancer drugs: improved drug solubilization and enhanced in vitro cytotoxicity. Eur. J. Pharm. Biopharm. 70, 51-57. doi: 10.1016/j.ejpb.2008.04.016

Schrama, D., Reisfeld, R. A., and Becker, J. C. (2006). Antibody targeted drugs as cancer therapeutics. Nat. Rev. Drug Discov. 5, 147-159. doi: 10.1038/nrd1957

Senthilkumar, R., Karaman, D. S., Paul, P., Björk, E. M., Odén, M., Eriksson, J. E., et al. (2015). Targeted delivery of a novel anticancer compound anisomelic acid using chitosan-coated porous silica nanorods for enhancing the apoptotic effect. Biomater. Sci. 3, 103-111. doi: 10.1039/c4bm00278d
Tae, H. S., Hines, J., Schneekloth, A. R., and Crews, C. M. (2010). Total synthesis and biological evaluation of tyroscherin. Org. Lett. 12, 4308-4311. doi: 10.1021/ol101801u

Weiner, S. J., Kollamn, P. A., Case, D. A., Singh, U. C., Ghio, C., Alagona, G., et al. (1984). A new force field for molecular mechanical simulation of nucleic acids and proteins. J. Am. Chem. Soc. 106, 765-784. doi: 10.1021/ja00315a051

Wen, X. S., Li, D., Zhang, Y. Y., Liu, S. P., Ghali, L., and Iles, R. K. (2012). Arsenic trioxide induces cervical cancer apoptosis, but specifically targets human papillomavirus-infected cell populations. Anticancer Drugs 23, 280-287. doi: 10.1097/CAD.0b013e32834f1fd3

Wu, C. C., Li, T. K., Farh, L., Lin, L. Y., Lin, T. S., Yu, Y. J., et al. (2011). Structural basis of type II topoisomerase inhibition by the anticancer drug etoposide. Science 333, 459-462. doi: 10.1126/science.1204117

Wu, X., and Ojima, I. (2004). Tumor specific novel taxoid-monoclonal antibody conjugates. Curr. Med. Chem. 11, 429-438. doi: 10.2174/0929867043455963

Zhang, Z. J., Tian, J., Wang, L. T., Wang, M. J., Nan, X., Yang, L., et al. (2014). Design, synthesis and cytotoxic activity of novel sulfonylurea derivatives of podophyllotoxin. Bioorg. Med. Chem. 22, 204-210. doi: 10.1016/j.bmc.2013.11.035

Zi, C. T., Yang, D., Dong, F. W., Li, G. T., Li, Y., Ding, Z. D., et al. (2015). Synthesis and antitumour activity of novel per-butyrylated glycosides of podophyllotoxin and its derivatives. Bioorg. Med. Chem. 23, 1437-1446. doi: 10.1016/j.bmc.2015.02.021

Zi, C. T., Yang, L., Gao, W., Li, Y., Zhou, J., Ding, Z. T., et al. (2017). Click glycosylation for the synthesis of 1,2,3-triazole-linked picropodophyllotoxin glycoconjugates and their anticancer activity. ChemistrySelect 2, 5038-5044. doi: $10.1002 /$ slct. 201700347

Conflict of Interest Statement: The authors declare that the research was conducted in the absence of any commercial or financial relationships that could be construed as a potential conflict of interest.

Copyright (c) 2019 Zi, Gao, Yang, Feng, Huang, Sun, Jin, Xu, Dong, Li, Ding, Zhou, Jiang, Yuan and Hu. This is an open-access article distributed under the terms of the Creative Commons Attribution License (CC BY). The use, distribution or reproduction in other forums is permitted, provided the original author(s) and the copyright owner(s) are credited and that the original publication in this journal is cited, in accordance with accepted academic practice. No use, distribution or reproduction is permitted which does not comply with these terms. 